\title{
Biomarkers Derived from Nicotine and its Metabolites: A Review*
}

\author{
by \\ Anthony R. Tricker \\ Philip Morris Products S.A., PMI Research and Development, CH-2000 Neuchâtel, Switzerland
}

\section{SUMMARY}

Nicotine is the major alkaloid present in tobacco and the most frequently determined compound as a biomarker of tobacco exposure in both smokers and non-smokers exposed to environmental tobacco smoke. Current knowledge on the human metabolism and disposition kinetics of nicotine is reviewed, together with methods for the determination of nicotine and various metabolites in different human biological fluids and matrices. Only short-term biomarkers of nicotine exposure exist and long-term biomarkers of exposure such as the incorporation of nicotine and cotinine into human hair, toenails and deciduous teeth require further investigation. Determination of "nicotine boost", the difference in blood nicotine concentrations that occur after smoking a single cigarette, provides an experimental indication of individual smoking behaviour, but is unsuitable for population studies. The determination of nicotine plus multiple phase I and phase II metabolites in 24-hour urine, often expressed as "nicotine equivalents", provides the most accurate way to determine exposure to nicotine in smokers; however, few laboratories are equipped to perform the complex analysis required for this purpose. Nicotine equivalents can be used to estimate the uptake of nicotine from a cigarette in both individuals and in population studies. Despite recent advancements in analytical methodology and the possibility of determining multiple nicotine metabolites in various biological fluids, determination of cotinine, the major metabolite of nicotine, is likely to remain the most commonly used approach to assess exposure to tobacco smoke in both smokers and non-smokers. Representative data for cotinine in blood, saliva and urine of smokers and non-smokers are presented. [Beitr. Tabakforsch. Int. 22 (2006) 147-175]

\section{ZUSAMMENFASSUNG}

Nikotin ist das Hauptalkaloid im Tabak und die am häufigsten als Biomarker zur Bestimmung der Tabakexposition von Rauchern und Nichtrauchern durch Tabakrauch in der Raumluft (ETS) analysierte Substanz. Die Arbeit beschreibt den gegenwärtigen Kenntnisstand über den Nikotin-Metabolismus im menschlichen Organismus und die Dynamik der Disposition sowie die Methoden zur Bestimmung von Nikotin und seiner Metaboliten in verschiedenen biologischen Matrizes des Menschen. Es existieren zur Zeit nur Kurzzeit-Biomarker der Nikotinexposition, während Langzeit-Biomarker wie die Einlagerung von Nikotin und Cotinin in menschliche Haare, Fußnägel und Milchzähne weiterer Untersuchungen bedürfen. Die Bestimmung des „Nikotinschubs”, der Zunahme der Nikotinkonzentration im Blut nach dem Rauchen einer einzelnen Zigarette, liefert einen experimentellen Hinweis auf das individuelle Rauchverhalten, es ist jedoch zur Untersuchung größerer Populationen ungeeignet. Die Bestimmung von Nikotin und mehrerer Metaboliten der Phase I und II im 24-h-Urin, was häufig als „Nikotinäquivalente” bezeichnet wird, ist das präziseste Verfahren zur Bestimmung der Nikotinexposition von Rauchern; nur wenige Labors verfügen jedoch über die notwendige Ausstattung zur Durchführung der hierfür erforderlichen komplexen Analysen. Nikotinäquivalente können dazu dienen, die Aufnahme von Nikotin durch eine Zigarette sowohl bei Individuen als auch in Studien mit größeren Populationen abzuschätzen. Trotz neuerer Fortschritte bei der analytischen Methodik und der Möglichkeit, eine Vielzahl von Nikotinmetaboliten in den verschiedenen biologischen Flüssigkeiten zu bestimmen, bleibt die Bestimmung von Cotinin, dem wichtigsten Nikotinmetaboliten, wohl das am 
häufigsten genutzte Verfahren zur Bestimmung der Tabakrauchexposition von Rauchern und Nichtrauchern. Repräsentative Daten für Cotinin im Blut, Speichel und Urin von Rauchern und Nichtrauchern werden präsentiert. [Beitr. Tabakforsch. Int. 22 (2006) 147-175]

\section{RESUME}

La nicotine est le principal alcaloïde présent dans le tabac et le composant le plus fréquemment déterminé comme biomarqueur de l'exposition au tabac des fumeurs et nonfumeurs exposés à la fumée de tabac ambiante. Cette revue présente les connaissances actuelles dans le domaine du métabolisme et la dynamique humaine pour la nicotine, de même que des méthodes de détermination de la nicotine et des divers métabolites dans les fluides et matrices biologiques humains. Ils existent seulement des biomarqueurs de l'exposition à la nicotine à court terme, les biomarqueurs à long terme comme l'incorporation de la nicotine et de la cotinine dans les cheveux humains, les ongles et les dents de lait nécessitent la réalisation d'études complémentaires. La détermination de la « poussée de nicotine », la différence des concentrations sanguines de la nicotine après fumage d'une cigarette, fournit une indication expérimentale du comportement individuel au fumage, mais cette méthode n'est pas appropriée pour des études portant sur une grande population. La détermination de la nicotine et les métabolites des phases multiples I et II dans l'urine de 24 heures, souvent nommée « équivalents de nicotine » fournissent l'approche la plus précise pour déterminer l'exposition à la nicotine des fumeurs ; cependant, peu de laboratoires disposent d'un équipement permettant de mener de telles analyses complexes. Des équivalents de nicotine peuvent servir à estimer l'absorption de la nicotine par une cigarette chez les individus de même que pour une grande population. Malgré des progrès récents dans la méthodologie analytique et la possibilité de déterminer de multiples métabolites de la nicotine dans les fluides biologiques, la détermination de la cotinine, principal métabolite de la nicotine, semble rester l'approche la plus souvent utilisée pour déterminer l'exposition à la fumée de tabac chez les fumeurs et non-fumeurs. Les données représentatives de la cotinine sanguine, de la salive et de l'urine des fumeurs et non-fumeurs sont présentées. [Beitr. Tabakforsch. Int. 22 (2006) 147-175]

\section{INTRODUCTION}

The validity of self-reports of smoking has often been questioned based on the belief that smokers tend to underestimate the actual amount smoked, or misclassify true smoking status as a consequence of social pressures to either reduce or quit smoking (1-4). The magnitude of misclassification of smoking status is evident from a review of 35 studies relating to denial of current smoking in which cotinine, a metabolite of nicotine, was determined in serum, saliva or urine as a biomarker of exposure to nicotine: $0.5-17.4 \%$ of self-reported non-smokers had cotinine concentrations consistent with current smoking, and $0.9-26.4 \%$ of true smokers with high concentrations of cotinine claimed to be non-smokers (5). Other commonly used biochemical measures of both active smoking and exposure to environmental tobacco smoke (ETS) include the determination of thiocyanate in blood, saliva and urine, carboxyhemoglobin $(\mathrm{COHb})$, and carbon monoxide in exhaled breath $(1,6)$. In a comparison of 11 different measures of smoke exposure (nicotine, cotinine and thiocyanate in plasma, saliva and urine, expired $\mathrm{CO}$ in exhaled breath, and $\mathrm{COHb}$ measurements), cotinine concentrations in plasma, saliva or urine proved to be the best indicator of current smoking status (1).

Nicotine per se and nicotine-derived metabolites in biological fluids are often used for validation of self-reported smoking status in clinical research $(1,7-10)$, investigation of changes in smoking behaviour (11), assessment of nonsmoker exposure to ETS (12-17), monitoring of nicotine uptake during nicotine replacement therapy (NRT) (18-23), and determination of compliance in subjects during smoking cessation $(3,24-26)$. Although there are some minor dietary sources of nicotine, these are insignificant compared to tobacco use $(9,27,28)$.

This review covers the current understanding of nicotine metabolism and pharmacokinetics in man and the use of different analytical techniques to determine nicotine and its metabolites as biomarkers of tobacco smoke exposure in smokers and non-smokers exposed to ETS.

\section{ABSORPTION OF NICOTINE FROM MAINSTREAM CIGARETTE SMOKE}

Nicotine in tobacco is found mainly as the levorotary $(S)$ isomer and only about $0.1-0.6 \%$ is present as $(R)$-nicotine (29). $(R)$-Nicotine accounts for up to $10 \%$ of total nicotine present in tobacco smoke, and presumably results from racemization of $(S)$-nicotine during tobacco combustion (30). Nicotine is distilled from burning tobacco and is present mainly in the particulate phase of mainstream cigarette smoke. Since mainstream cigarette smoke, the smoke inhaled by the smoker, contains small particles (mass mean aerodynamic diameter $<0.5 \mu \mathrm{m}$ ) they would be expected to penetrate the small airways and alveolar region of the lung. Mainstream cigarette smoke from cigarettes made mainly from flue-cured tobacco is slightly acidic (around $\mathrm{pH} 5.5$ ) resulting in limited absorption of nicotine through the oral mucosa into the systemic circulation (31-37). In contrast to this, about $90 \%$ of nicotine in cigarette smoke is retained at shallow inhalations as low as $75 \mathrm{~mL}$ (34). At deeper inhalations when cigarette smoke reaches the small airways and alveoli of the lungs, almost quantitative absorption of nicotine occurs regardless of the $\mathrm{pH}$ of smoke (38), resulting in concentrations of $20-100 \mathrm{ng}$ nicotine $/ \mathrm{mL}$ arterial blood (39-41). Rapid pulmonary absorption of nicotine from cigarette smoke is presumably due to the large alveolar surface area, thin alveolar endothelial layer, dissolution of nicotine into fluid of physiologic $\mathrm{pH}$ covering the alveolar surface, an extensive capillary bed, and a large blood flow.

Nicotine uptake is determined mainly by individual smoking patterns (42-44). Nicotine concentrations in body fluids vary widely among individuals, but are rather constant for an individual (43). Wide interindividual variation is observed even when smoking the same number of identical 
cigarettes (45). Consequently, the cigarette smoking machine yield of nicotine obtained from machine-smoking of cigarettes under standardized conditions, e.g., according to Federal Trade Commission (FTC) and International Standards Organization (ISO) conditions, is a poor predictor of the human smoker yield and uptake of nicotine from cigarette smoke (46-66). The results of standardized cigarette smoking machine methods do not represent the full behavioural range of individual smokers; as the FTC itself recognized, they simply indicate the relative yield position of brands according to a convention of analytical standards, but not actual conditions of smoking topography or smoke intake (67). Smokers who switch from higher-yield to a lower-yield cigarette have a higher nicotine exposure than is predicted by machine-smoking of cigarettes according to standardized conditions; conversely, smokers when they switch from a lower yield cigarette to a higher-yield cigarette have a lower exposure to nicotine than predicted (reviewed in 11).

Over the past 25 years cigarette smoking machine yields of 'tar' and nicotine for commercial cigarettes in most countries have gradually decreased. The sales-weighted mean 'tar' and nicotine yields under ISO smoking conditions of manufactured filter cigarettes in the UK have decreased from $19.0 \mathrm{mg}$ 'tar' and $1.23 \mathrm{mg}$ nicotine in 1972 to $9.5 \mathrm{mg}$ 'tar' and $0.79 \mathrm{mg}$ nicotine in 1999 (15). Over the same time period, sales-weighted average 'tar' and nicotine yields of plain non-filter cigarettes have decreased from 28.6 to $12.5 \mathrm{mg}$ 'tar' and 1.86 to $0.96 \mathrm{mg}$ nicotine/cigarette, respectively. Similar decreases in sales-weighted average 'tar' and nicotine yields per cigarette have been observed over the same time period in Japan (68) and the US (69). As a consequence of the reductions in nicotine yield determined by machine-smoking of cigarettes under standardized conditions over the last 25 years, care has to be taken in evaluating early reports of nicotine and nicotine-derived metabolite concentrations in biological fluids since these may not be representative of exposure to nicotine from current products.

Several studies have tried to estimate the dose of nicotine absorbed from a cigarette. Under unrestricted smoking conditions with subjects smoking their preferred brand of cigarettes, BENOWITZ and JACOB (52) used measurements of nicotine plasma clearance and mean steady-state plasma concentrations of nicotine, or area under the blood concentration-time curve following smoking compared to the area under the blood concentration-time curve of a known intravenous dose of nicotine, to estimate an average absorbed dose of $1.04 \pm 0.36 \mathrm{mg}$ nicotine/cigarette for cigarettes with a yield of $1.24 \pm 0.27 \mathrm{mg}$ nicotine/cigarette (range, 0.87-1.80) under FTC smoking conditions. The same working group has also estimated an average uptake of $2.29 \pm 1.00 \mathrm{mg}$ nicotine/cigarette for cigarettes with a cigarette smoking machine yield of $1.1 \pm 0.2 \mathrm{mg}$ nicotine under FTC smoking conditions (70), and an average uptake of $0.87 \mathrm{mg}$ nicotine/cigarette (range, 0.22-1.92) for cigarettes with a cigarette smoking machine yield of $1.0 \pm$ $0.2 \mathrm{mg}$ nicotine under FTC smoking conditions (71). Using a similar approach, the average dose of nicotine absorbed per cigarette has been estimated as $1.06 \mathrm{mg}$ nicotine; $82 \%$ of the cigarette smoking machine yield of $1.3 \mathrm{mg}$ nicotine (53). Nicotine uptake per cigarette shows ethnic differ- ences $(72,73)$, which may, in part, be related to ethnic differences in nicotine clearance (72). In a study of 40 African-American and 39 Caucasian smokers, nicotine uptake per cigarette was 30\% higher in African-American compared to Caucasian smokers (1.41 vs. $1.09 \mathrm{mg}$ nicotine/cigarette; $p=0.02$ ) for cigarettes of similar cigarette smoking machine yields of nicotine (mean: $1.1 \mathrm{vs} .1 .0 \mathrm{mg}$ nicotine/cigarette) under FTC smoking conditions (72).

Nicotine uptake per cigarette has been estimated from cotinine concentrations in saliva (64). Estimates of nicotine uptake per cigarette varied from $1.07 \mathrm{mg}$ nicotine for cigarettes with a cigarette smoking machine yield of $0.1 \mathrm{mg}$ nicotine under ISO smoking conditions, $1.17 \mathrm{mg}$ nicotine ( $<0.4 \mathrm{mg}$ ISO nicotine/cigarette), $1.22 \mathrm{mg}$ nicotine $(0.4-0.8$ $\mathrm{mg}$ ISO nicotine/cigarette), to $1.31 \mathrm{mg}$ nicotine $(>0.8 \mathrm{mg}$ ISO nicotine/cigarette) suggesting only a slight tendency for smokers of cigarettes with a higher nicotine yield to have higher nicotine uptake per cigarette. For cigarettes with a nominal cigarette smoking machine yield of $1 \mathrm{mg}$ nicotine under ISO smoking conditions the estimated uptake was $1.4 \mathrm{mg}$ nicotine/cigarette.

The absorbed dose of nicotine per cigarette has also been estimated from the amount of nicotine metabolites excreted in $24 \mathrm{~h}$ urine $(44,62,63,74)$. Based on the ratio of $24 \mathrm{~h}$ urinary excretion of nicotine equivalents (sum of nicotine plus seven nicotine-derived metabolites) over cigarette smoking machine yield of nicotine under ISO smoking conditions multiplied by the number of cigarettes smoked per day, ANDERSSON et al. (62) estimated a mean dose of $1.32 \pm 0.54 \mathrm{mg}$ nicotine/cigarette for 143 smokers of cigarettes with a mean yield of $1.10 \pm 0.23 \mathrm{mg}$ nicotine/cigarette under ISO smoking conditions. In a series of studies, BYRD et al. (74) reported that the sum of nicotine plus eight metabolites in $24 \mathrm{~h}$ urine collected from 11 smokers was very close $(96 \pm 29 \%$, range $58-144 \%)$ to the cigarette smoking machine yields of nicotine under FTC smoking conditions for the total number of cigarettes smoked over the same period. In a larger study of 33 smokers, individual variability (CVs of 0.39-0.80) in nicotine uptake per cigarette in different FTC nicotine bands (0.13-0.14, 0.42-0.56, 0.62-0.82, and 0.96-1.30 mg FTC nicotine/cigarette) suggested that nicotine uptake was a function of individual smoking behaviour within different product design (i.e., nicotine yield) limits (44). In a repeat study using 113 smokers of which only 72 subjects demonstrated reasonable compliance with the study protocol, cigarette smoking machine yields of nicotine under FTC smoking conditions was only weakly related to nicotine uptake, and individual smoking behaviour exerted a greater influence on the amount of nicotine uptake (63).

\section{METABOLISM OF NICOTINE}

Nicotine is extensively metabolised in the liver, and to a small extent in the lung and kidney. The major pathways of nicotine metabolism are summarized in Figure 1. Nicotine is metabolized primarily by $\mathrm{C}$-oxidation to cotinine, and to a lesser extent by $\mathrm{N}$-oxidation to nicotine $\mathrm{N}$-1'-oxide, $\mathrm{N}$ demethylation, and $\mathrm{N}$-glucuronidation. Cotinine is further metabolized by hydroxylation to trans-3'-hydroxycotinine and 5'-hydroxycotinine, $\mathrm{N}$-oxidation to cotinine $\mathrm{N}$-1-oxide, 
and $N$-glucuronidation. The compound trans-3'-hydroxycotinine is further metabolized by $O$-glucuronidation. Glucuronides of nicotine, cotinine, and trans-3'-hydroxycotinine are major urinary metabolites of nicotine in man (75). These glucuronides have been structurally characterized as $(S)-(-)$ nicotine- $N$-1- $\beta$-D-glucuronide (76), $(S)-(-)$-cotinine- $N-1-\beta$ D-glucuronide (77), and trans-3'-hydroxycotinine- $O-\beta$-Dglucuronide (78). Several additional minor urinary metabolites have also been identified which probably account for less than $10 \%$ of total nicotine metabolism (79).

\section{Pharmacokinetics of nicotine and its major metabolites}

The pharmacokinetics of nicotine, cotinine, and trans-3'hydroxycotinine are well characterized in man $(71,73$, 80-89). Conflicting studies report that the metabolic clearance of plasma nicotine is higher in smokers than in nonsmokers (82); however, other studies find slightly lower clearance in smokers compared to non-smokers $(86,90)$. The observed discrepancy may be due to the use of racemic nicotine in one study $(82)$ rather than $(S)$-nicotine used in the other two studies $(86,90)$. Clearance of nicotine normalized for body weight is significantly slower in smokers compared to non-smokers, and cotinine clearance is similar in smokers and non-smokers (90). Lower nicotine clearance was attributed to inhibition of nicotine metabolism by some unidentified component(s) of tobacco smoke (86). The disposition pharmacokinetics of nicotine and metabolism are dose-independent (90). Total and nonrenal clearance of nicotine is, on average, slightly higher in Caucasians than in African-Americans (72) and ChineseAmericans (73) and decreased in subjects with either alcohol-induced liver cirrhosis (91) or kidney failure (92). On average, $70 \%$ to $80 \%$ of nicotine is converted to cotinine prior to metabolism to other metabolites $(71,93)$.

Intravenous infusion experiments with abstinent adult smokers show mean plasma elimination half-lives of $2.3 \mathrm{~h}$ (range, 1.6-2.8 h) for nicotine (71), $17.5 \mathrm{~h}$ (range, 8.1-29.3 h) for cotinine (71), and $6.6 \mathrm{~h}$ (range, 4.6-8.3 h) for trans-3'-hydroxycotinine (87). No statistically significant differences are apparent in the disposition of nicotine in men and women $(71,72)$, and in elderly subjects compared to younger adults (94). The clearance of nicotine and cotinine is significantly higher $(60 \%$ and $140 \%$, respectively), and the half-life of cotinine much shorter $(8.8 \mathrm{~h})$, during pregnancy compared to postpartum (95). The mean blood half-lives of nicotine and cotinine in newborns are $11.2 \mathrm{~h}$ and $16.3 \mathrm{~h}$, respectively (96).

The mean elimination half-lives in newborns are 9.0, 22.8, and $18.8 \mathrm{~h}$ for nicotine, cotinine and trans-3'-hydroxycotinine; and 13.0, 19.8, and $19.4 \mathrm{~h}$ for conjugated nicotine, conjugated cotinine and conjugated trans-3'-hydroxycotinine, respectively (96). The average urinary elimination half-life for conjugated trans-3'-hydroxycotinine in adult smokers is $7.2 \mathrm{~h}$ (range, 4.6-9.4 h) (87). The fractional conversion of nicotine to cotinine, the metabolic clearance of nicotine to cotinine, and the clearance of cotinine are significantly lower in African-Americans compared to Caucasians (88). African-Americans excrete significantly less nicotine as nicotine- $N$-glucuronide and less cotinine as cotinine- $N$-glucuronide than Caucasians, but there is no difference in the excretion of trans-3'- hydroxycotinine- $O$-glucuronide. The extent of both $\mathrm{N}$ - and $O$-glucuronidation shows a high interindividual variability in both adults $(44,62,63,74,88,93,97,98)$ and newborns (96). In both smokers and subjects using transdermal nicotine, the extent of $\mathrm{N}$-glucuronidation of nicotine and cotinine is highly correlated, but neither is correlated with the extent of $O$-glucuronidation of trans-3'-hydroxycotinine (93). Menthol, used as an additive in cigarettes, inhibits nicotine metabolism by slower oxidation to cotinine and by slower $N$-glucuronide conjugation, but does not substantially affect cotinine metabolism (99).

\section{Genetic influence on nicotine metabolism}

Several cytochrome P450 (CYP) enzymes have been identified which mediate in vitro mammalian metabolism of nicotine to cotinine (100-103). It is generally accepted that CYP2A6 is the major CYP enzyme responsible for in vivo hepatic C-oxidation of physiological concentrations of nicotine (102-105) and C-hydroxylation of cotinine to trans-3'hydroxycotinine (106). Although CYP2B6 and CYP2D6 mediate in vitro nicotine C-oxidation $(100,101)$, neither is thought to be involved in the metabolism of physiological concentrations of nicotine $(105,107)$. CYP2A13 has considerable activity towards both nicotine and cotinine (103) and its role in nicotine metabolism in extrahepatic tissues remains to be fully clarified. All four CYP enzymes are highly polymorphic (108-111). Flavin-containing monooxygenase 3 (FMO3) is the main enzyme responsible for nicotine- $\mathrm{N}-1$ 'oxide formation (112). The enzyme(s) responsible for the metabolism of nicotine to nornicotine and conversion of cotinine to cotinine- $N$-1-oxide are unknown. Preliminary studies suggests that UDP-glucuronosyltransferases UGT1A4 and UGT1A9 catalyse both nicotine and cotinine $\mathrm{N}$-glucuronidation (113).

It has been proposed that genetic polymorphisms may influence nicotine metabolism and contribute to differences in an individual's smoking behaviour $(114,115)$. Thus, polymorphism of the CYP2A6 locus associated with reduced enzyme activity would be expected to result in compromised depletion of plasma nicotine concentrations and, as a consequence, reduced smoking behaviour. This hypothesis is based on the premise that a smoker engages in smoking behaviour in such a way as to maintain plasma nicotine concentrations within a constant range (116). In support of this hypothesis, an experimental study reports that the metabolic ratio of total trans-3'-hydroxycotinine (trans-3'-hydroxycotinine plus its glucuronide conjugate):total cotinine (cotinine plus its glucuronide conjugate), which may reflect the rate of metabolism of cotinine to trans-3'-hydroxycotinine and hence CYP2A6 activity in smokers, is significantly correlated with the number of cigarettes smoked per day $(r=0.33 ; p=0.005)$ (117). However, contrary to this observation, population studies fail to consistently and conclusively demonstrate any associations between specific variant $C Y P 2 A 6$ alleles encoding for either reduced or enhanced enzyme activity with selfreported smoking behaviour (110). It is unlikely that either nicotine $\mathrm{N}$-oxidation or phase II metabolism of nicotine to nicotine $N$-glucuronide significantly contributes to blood nicotine depletion as these metabolites are quantitatively only minor metabolites of nicotine (Figure 1). 


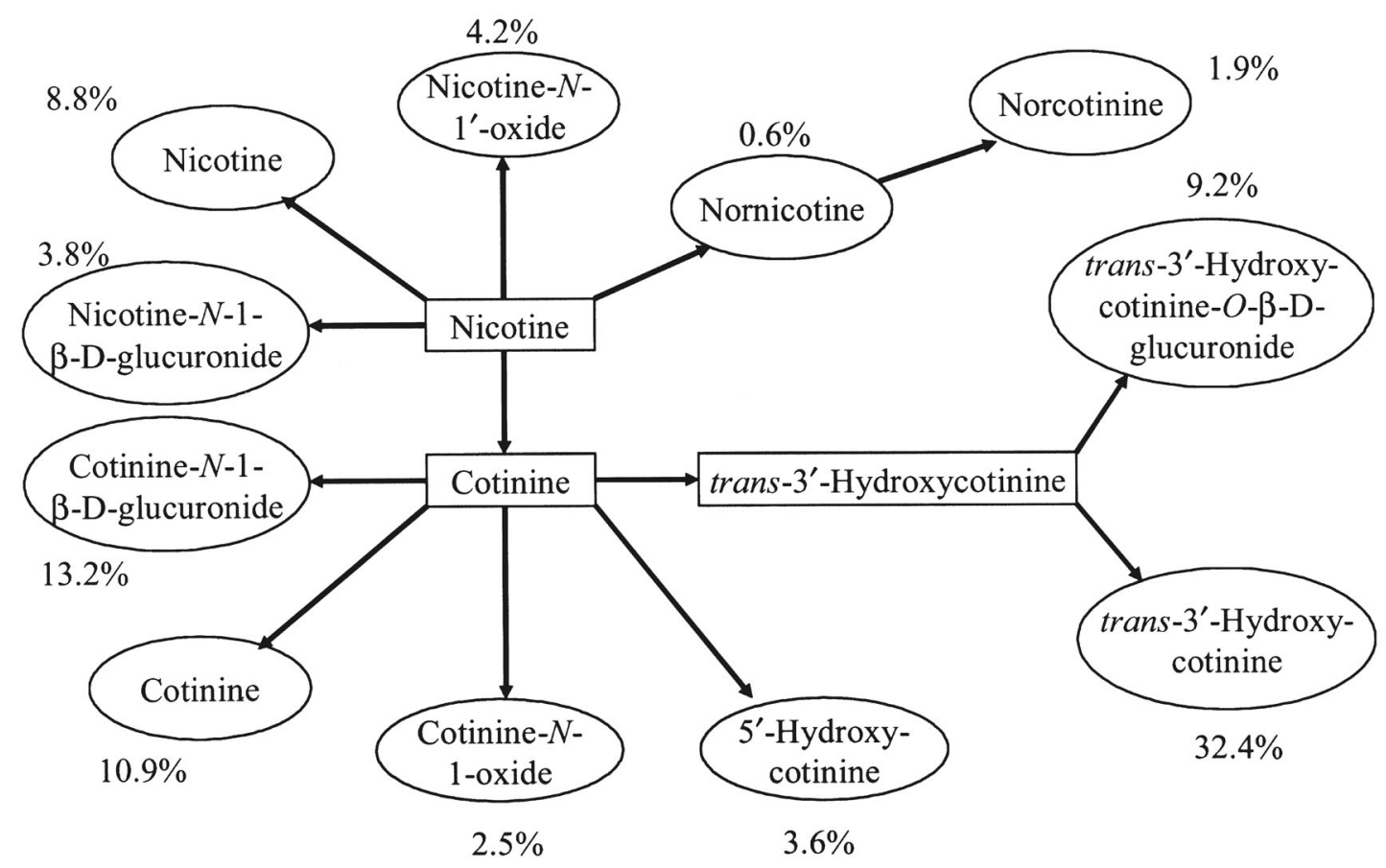

Figure 1. Quantitative scheme of nicotine metabolism, based on average excretion of major nicotine metabolites as percentage of total urinary nicotine $(62,74,93,97,98,323)$

\section{ANALYTICAL METHODS FOR THE DETERMINATION OF NICOTINE AND NICOTINE-DERIVED METABOLITES}

Nicotine and the major nicotine-derived metabolites can be measured in various biological media (118). The most common techniques are based on colorimetric methods (119), immunoassays (120), gas chromatography (GC) (121), and high-performance liquid chromatography (HPLC) (122). Each of these techniques has its advantages and disadvantages, and care must be taken in comparing results obtained in one laboratory with those obtained in another laboratory (123). A general concern in the analysis of nicotine, but not other nicotine-derived metabolites, is inadvertent background contamination of samples and laboratory equipment by nicotine (124). Although several laboratories have reported procedures to limit the possibility of laboratory contamination (124-128), constant surveillance is required to avoid probable sources of nicotine contamination.

\section{Colorimetric assays}

Colorimetric assays, based on the König reaction, involve the derivatisation of nicotine and nicotine-derived metabolites containing an unsubstituted pyridine nitrogen with either barbituric acid or barbituric acid derivatives, e.g., 1,3-diethyl-2-thiobarbituric acid (DETBA), to form coloured chromophores $(119,129)$. In the direct barbituric acid (DBA) assay (129), a positive test result is indicated by the development of an orange colour within $20 \mathrm{~min}$. Quantitative determination of nicotine metabolites is made by determination of the optical density of the sample at $506 \mathrm{~nm}$ compared to the colour developed by a standard solution of $10 \mu \mathrm{g}$ cotinine/mL water, and results expressed as " $\mu \mathrm{mol} / \mathrm{L}$ cotinine equivalents". The results obtained using the DBA assay have also been referred to as the "Barlow index" (61). In the DETBA assay (129), a positive test result is indicated by the development of a pinkred colour within $20 \mathrm{~min}$. The DETBA assay can be improved by extraction of the coloured chromophore(s) into ethyl acetate $(0.5 \mathrm{~mL})$ and determination of the optical density at $532 \mathrm{~nm}$ compared to the colour developed by a standard solution of $10 \mu \mathrm{g}$ cotinine/mL water. Both condensation with barbituric acid or DETBA provide nonspecific methods for the qualitative determination of smoking status since endogenous compounds present in urine may cause false-positive test results $(57,130)$. In addition, the instability of the chromophores may result in a falsenegative response (130-133). A number of rapid screening tests have been developed to evaluate current smoking status using colorimetric assays (57, 58, 134-136).

HPLC using either diode array detection (DAD) or ultraviolet (UV) detection has been used to quantify individual nicotine metabolite chromophores formed by barbituric acid $(57,130)$ and DETBA $(132,133,137,138)$. Carefully controlled derivatisation and injection conditions are required for the analysis of DETBA derivatives (133) since the derivatives are unstable unless extracted into organic solvents, such as butanol, ethyl acetate and chloroform $(132,137)$. HPLC-DAD analysis of DETBA chromophores allows the determination of nicotine plus 12 metabolites in rodent and human urine (138). The sensitivity of the DETBA assay can be further enhanced by using the 1,3-dibutyl-2-thiobarbituric acid (DBTBA) analogue and extraction of the chromophores into ethyl acetate prior to HPLC-UV analysis (139).

Despite the unspecific nature of colorimetric assays, urinary "cotinine equivalents" correlate well with daily cigarette consumption $(57,58,61,136)$. However, a correlation and discrimination between light and heavy smokers is 
not apparent in all studies $(129,130)$. The results obtained by colorimetric assays for "cotinine equivalents" in urine correlate well with the results obtained for determination of cotinine concentrations by radioimmunoassay (RIA) $(119,131,134), \operatorname{GC}(61,136)$, and $\operatorname{HPLC}(57,58,130)$. However, in all cases, colorimetric assay results for "cotinine equivalents" are consistently and quantitatively 3 - to 4-fold higher than the corresponding results for cotinine concentrations in urine determination by RIA, GC or HPLC methods.

\section{Immunoassays}

Immunoassay techniques used for the determination of nicotine and cotinine concentrations in biological media include RIA (140-142), enzyme-linked immunosorbent assay (ELISA) (143-145), and fluorescence immunoassay (FIA) $(135,143,146)$. Cotinine has also been determined using enzyme immunoassay (EIA) $(147,148)$. Immunoassays have been used to determine nicotine concentrations in amniotic fluid (149), blood plasma and serum (150-155), cervix mucus $(153,156)$, hair (157-159), meconium (160), saliva (154), seminal plasma (155), and urine $(154,161)$. Cotinine concentrations have been determined by immunoassays in amniotic fluid $(149,162)$, blood plasma and serum $(17,145$, $153-155,163-166)$, cervix mucus $(153,156)$; follicular fluid (167-169), hair (157-170), saliva $(144,145,154)$, seminal plasma $(155,165,171,172)$, and urine $(12,134,144,148$, 154, 161, 165). The International Agency for Research on Cancer (IARC) has often recommended a RIA developed for nicotine and cotinine (141) for exposure assessment of these metabolites in blood, saliva, and urine $(154,173)$.

Monoclonal antibodies raised against cotinine show minimal cross-reactivity to nicotine-derived metabolites and structurally-related compounds $(140,141,144,163)$. Monoclonal antibody methods for the determination of cotinine usually agree well with chromatographic methods, although it is advisable to seek confirmation of results obtained by immunoassays using an independent method of analysis (174). Cotinine concentrations in saliva, serum and plasma determined using monoclonal antibody-based ELISA and fluorescence immunoassays show strong correlations with cotinine concentrations obtained by both RIA and GC (163). Strong correlations also exist between cotinine concentrations in plasma determined by RIA and HPLC-UV detection (164), and nicotine concentrations in urine determined by EIA and GC-MS (148).

Polyclonal anti-cotinine sera raised in rabbits (140) shows a relative cross-reactivity of about $30 \%$ to (+)-trans-3'hydroxycotinine, $11.5 \%$ to $(+)$-cis-3'-hydroxycotinine, and $6.5 \%$ to $(+)$-demethylcotinine, but no appreciable crossreactivity to nicotine or other nicotine metabolites (175, 176). Since trans-3'-hydroxycotinine is present in urine at concentrations higher than cotinine (Figure 1), polyclonal antibodies tend to overestimate the cotinine concentration in urine (by as much as ca. 2.9 fold) compared to chromatographic methods $(161,177)$.

\section{Gas chromatography}

Gas chromatography (GC) methods are well established for the quantitation of nicotine, cotinine and trans-3'- hydroxycotinine in biological media (11). Initially either flame ionization detectors (FID) or more selective nitrogen-phosphorus detectors (NPD) were the detectors of choice $(124,125,127,178-186)$. Most laboratories now use GC interfaced to a mass spectrometer (MS) which provides a more sensitive and specific method for the measurement of nicotine and multiple nicotine-derived metabolites (121). A number of simple bench-top capillary GCMS methods using selected ion monitoring have been developed for the determination of nicotine $(126,187,188)$, cotinine $(148,161,189-192)$, and trans-3'-hydroxycotinine (182) in various biological matrices. Methods have also been developed for the simultaneous analysis of nicotine and cotinine (50,128, 193-195), and the simultaneous analysis of nicotine, cotinine, and trans-3'-hydroxycotinine (186). Conjugated metabolites of nicotine, cotinine, and trans-3'-hydroxycotinine are determined as the difference in the total amount of nicotine, cotinine, and trans-3'hydroxycotinine before and after incubation with $\beta$-glucuronidase $(21,93)$. A GC-MS method for the simultaneous determination of nicotine, cotinine and thiocyanate concentrations in urine within a single run has also been developed (196). This method has also been applied to the determination of nicotine and cotinine concentrations in saliva.

\section{Liquid chromatography}

Liquid chromatography (LC) is more versatile than GC and can be applied to a greater variety of nicotine-derived metabolites since only metabolite dissolution in the mobile phase rather than volatilization is required. Nicotine has been determined by HPLC with electrochemical (EC) detection (197-199), while cotinine analysis is performed mainly by HPLC with UV detection prior to derivatisation $(58,164,200-202)$ or as the barbituric acid derivative (57). Simultaneous determination of nicotine and cotinine is possible using HPLC with DAD (203), and UV detection prior to derivatisation $(204,205)$ or after pre-column DETBA derivatisation (137).

The real advantage of using HPLC compared to GC separation is in its application to the simultaneous analysis of nicotine plus multiple nicotine metabolites. HPLC-DAD analysis of DETBA derivatives of nicotine plus 12 metabolites is reported in rodent and human urine (138). Glucuronide conjugates of nicotine, cotinine, and trans-3'-hydroxycotinine have been determined in urine as the difference in the total concentration prior to and after incubation with $\beta$-glucuronidase. Conjugated metabolites of cotinine and trans-3'hydroxycotinine in blood plasma have also been determined as free cotinine and trans-3'-hydroxycotinine following alkaline hydrolysis at $70{ }^{\circ} \mathrm{C}$ by HPLC-UV with detection at 254 $\mathrm{nm}$ (206). However, these methods may be subject to complicated extraction procedures required for sample preparation and derivatisation, low analyte recovery, long chromatographic run times, and low sensitivity.

Radiometric HPLC has been extensively used in both in vitro experiments and human studies of nicotine biotransformation, and to determine the mass balance of various metabolites after administration of labelled nicotine to laboratory animals $(207,208)$.

HPLC-MS has been used for the determination of nicotine, cotinine and trans-3'-hydroxycotinine concentrations in 
blood serum and seminal plasma (209). Determination of urinary nicotine, cotinine, trans-3'-hydroxycotinine, their glucuronide conjugates as aglycones, nicotine- $N$-1'-oxide, and cotinine- $N$-1-oxide has been reported using $\beta$-glucuronidase treatment of urine followed by HPLC separation of metabolites, reversed-gradient addition to the column eluent, and thermospray LC-MS (74). Reversed-gradient addition to the column eluent is required to provide a stable baseline, and to improve the reproducibility of the response to nicotine metabolites. The major advantage of thermospray LC-MS is that relatively polar and thermally unstable metabolites can be introduced into the mass spectrometer via the thermospray interface. Thermospray MS has been widely used for the determination of nicotine metabolites which lack thermal stability such as $\mathrm{N}$-oxides and glucuronide conjugates $(63,74,77,78,210,211)$.

A major advance in analytical technology over the last few years has been the analysis of nicotine metabolites by LC interfaced to a tandem mass spectrometer (LC-MS-MS) using improved atmospheric pressure ionization sources. Cotinine and trans-3'-hydroxycotinine concentrations in saliva can also be determined by automated solid-phase extraction and reversed-phase LC-MS-MS using an electrospray ionization interface $(212,213)$. Following solid-phase extraction of saliva, cotinine has also been determined by LC with an atmospheric pressure ionization interface to a tandem mass spectrometer (LC-API-MS-MS) (214). LC-API-MS-MS methods have been widely applied to the analysis of cotinine concentrations in blood serum $(14,214-216)$, and nicotine and cotinine concentrations in blood plasma $(217,218)$. Simultaneous analysis of nicotine, cotinine, trans-3'-hydroxycotinine, anabasine and nornicotine concentrations in human serum and urine is reported by solid-phase extraction and LC-API-MS-MS (219). Several methods report the analysis of nicotine plus multiple metabolites in urine using LC-API-MS-MS (98, 220-222). The limits of quantification (LOQ) for nicotine and its metabolites in these methods range from low $\mu \mathrm{g} / \mathrm{L}$ to $10-20 \mu \mathrm{g} / \mathrm{L}$ urine, which is sufficient for the determination of nicotine metabolites in clinical studies.

\section{Test strip assays}

Test strip or "dip-stick" assays (e.g., NicCheck I® and NicoMeter ${ }^{\circledR}$ ) provide a simple and inexpensive method for the detection of cotinine in urine as a screening tool to verify current smoking status in a non-laboratory environment. The NicCheck I® assay, is based on the König reaction with nicotine and nicotine-derived metabolites (129, 131) that has been adapted to test strip format. The test strip is immersed in urine and the colour reaction visually read by eye. However, similar to colorimetric assays, a number of other endogenous compounds and drug metabolites in urine contain an unsubstituted pyridine ring (e.g., nicotinic acid, isoniazid, nicotinamide) that may give rise to a false-positive test result (223). Not surprisingly, a poor correlation exists between cotinine concentrations in urine determined using the NicCheck I ${ }^{\circledR}$ test strip and confirmation by more specific GC-MS (223).

The NicoMeter® test strip is an immunoassay that allows semi-quantitative determination of cotinine and, to a lesser extent, trans-3'-hydroxycotinine. If used according to the manufacturer's instructions, the NicoMeter® test strip can be used for determination of cotinine in saliva and urine, and is claimed to be a valid method for confirming self-reported smoking status. The sensitivity and specificity are comparable to both ELISA and GC-MS methods (224, 225).

While test strip assays are often considered to be an attractive low cost screening method for determining current smoking status, several limitations have to be taken into consideration in order to minimize the number of falsepositive and false-negative results. Detection limits are poorly defined and over-estimation of cotinine concentrations occurs with both the NicCheck I® and NicoMeter ${ }^{\circledR}$ test strips. The high detection threshold makes these methods inappropriate for the determination of ETS exposure.

\section{Miscellaneous screening methods}

Confirmation of current smoking status has been evaluated by semi-quantitative determination of nicotine and cotinine in urine using thin layer chromatography (TLC) on silica gel $\mathrm{G}$ and visualization using an iodoplatinate spray (226), and more recently by combined solid-phase extraction followed by high-performance thin-layer chromatography (HPTLC) with visualization of cotinine using a ninhydrin/cadmium acetate monohydrate spray (227). Separation of nicotine and 10 metabolites is possible by capillary zone electrophoresis (CZE) and CZE-MS, although these methods generally lack sensitivity and specificity (228). To overcome the lack of sensitivity, 200-fold sample concentration by solid-phase extraction and sample stacking CZE-MS is reported for the identification of nicotine and eight metabolites in urine (229). However, these methods have seen little application in the analysis of biological matrices or clinical samples.

\section{NICOTINE AND NICOTINE-DERIVED METABOLITES IN BIOLOGICAL MATRICES}

The relatively short half-life of nicotine $\left(\mathrm{t}_{1 / 2} \sim 2.3 \mathrm{~h}\right)$ and large interindividual differences in the extent of nicotine metabolism to cotinine precludes its use as an accurate marker of nicotine uptake from cigarette smoke $(43,90,93)$. Since cotinine, the principal metabolite of nicotine, has a half-life of about $17 \mathrm{~h}$, it has been assumed to be a better biochemical measure of nicotine uptake in smokers and non-smokers (1, 13, 90). Cotinine concentrations in blood and saliva are highly correlated and the saliva to blood ratio is 1.1-1.4 (83, $84,194,200,214,230-233)$. The cotinine concentrations in blood and urine are also correlated (152) and the urine to blood ratio is ca. 5.0 (234). Thus, the cotinine concentration in blood can be estimated by determination of cotinine concentrations in either saliva or urine. As a consequence, the choice of which biological matrix to collect for determination of cotinine in any given study should depend on the objective of the study and on practical rather than pharmacokinetic considerations $(232,235,236)$. Although blood and saliva are considered the samples of choice for making quantitative assessments of tobacco smoke exposure and/or nicotine uptake (174), the convenience and non-invasiveness of urine sample collection makes this an attractive matrix for assessment of smoking status and exposure to ETS in epidemiological studies. 
Only a few studies have determined multiple biomarkers of tobacco smoke exposure in the same subjects $(1,52,54,55$, $59,83,84,200,214,230-234,237-239)$, or the same biomarker in different biological matrices of each subject (165, 236). JARVIS et al. (1) evaluated the predictive value of 11 different measures of smoke intake $(\mathrm{COHb}$ and exhaled $\mathrm{CO}$; nicotine, cotinine and thiocyanate in plasma, saliva and urine) to discriminate smokers from non-smokers and concluded that the concentration of cotinine, whether determined in plasma, urine or saliva, was the best indicator of current smoking. Several studies using multiple biomarkers of tobacco smoke exposure confirm that the use of nicotine and nicotine-derived metabolites as the most appropriate indicator of tobacco smoke exposure $(7,54,240)$.

In order to optimise the cut-off point used to discriminate between non-smokers and smokers, a receiver operating characteristic (ROC) curve of the decision threshold effect (241) should be constructed. A ROC curve is a simple empirical description of sensitivity, the true-positive fraction, against the complement of specificity, the false-positive fraction, for all possible cut-off values. Optimum cutoff values for biochemical validation of smoking status are dependent on the prevalence of smoking in the population tested (242). When the prevalence of smoking is low, the number of misclassifications depends primarily on the false-positive rate of the assay. Thus, the cut-off value needs to be higher to minimise the false-positive rate. Misclassification rates vary depending on the choice of cut-off value $(3,5,243)$, the specificity of the assay used to discriminate between smokers and non-smokers (161), the study population $(5,244)$, and the amount smoked within the smoking population $(244,245)$. Denial of current smoking is higher in self-reported ex-smokers than in nonsmokers (243), occasional smokers compared to regular smokers $(5,166)$, adolescents compared to the general population (2), and may vary with educational background (243) and between different ethnic populations (243, 246, 247). Possible reasons for misclassification include reporting error as a consequence of social pressures to either reduce or quit smoking (1-4), and the use of inappropriate cut-off values for delineation of smoking status (3). The sensitivity of any cut-off value is dependent on the time at which the subjects smoked their last cigarette $(71,239$, 244) or were exposed to ETS (234). Maximized sensitivity and selectivity of cut-off values may vary with ethnicity (3, $238,243,248,249)$, and may require defining for both pregnant and non-pregnant women $(95,250)$.

Compliance to NRT and avoidance of tobacco product use cannot be assessed using nicotine and nicotine-derived metabolite measurements; however, determination of minor tobacco alkaloids such as anabasine in biological matrices suggest that tobacco-related sources of nicotine may have been used in addition to NRT $(219,251,252)$.

\section{Blood}

In smokers, nicotine concentrations in blood increase over the first 4 to $6 \mathrm{~h}$ of the smoking day (range, 10 to $50 \mathrm{ng}$ nicotine/mL blood) and then tend to plateau until smoking is stopped $(53,127,253-255)$. The increment increase in blood nicotine concentration on smoking a single cigarette ranges from 5-30 ng nicotine/mL blood, depending on how the cigarette is smoked $(38,39,42,53,253,256)$. During the night, nicotine concentrations in blood decline and little nicotine is present in blood of smokers when they wake in the morning $(45,127)$. Consequently, the best time for collecting blood samples for the determination of nicotine and cotinine is in the mid afternoon at which time almost steady state conditions can be assumed (52). At normal physiological conditions, about $4.9 \pm 8.0 \%$ of nicotine (254) and $2.6 \pm 3.5 \%$ of cotinine (255) are bound to plasma proteins. The blood:plasma cotinine ratio is $0.88 \pm$ 0.02 , and the erythrocyte:unbound plasma concentration ratio $0.74 \pm 0.04$. Nicotine and cotinine are both stable in plasma samples stored at ambient temperature for 12 days (257). Blood serum cotinine is stable at $37{ }^{\circ} \mathrm{C}$ for up to 6 weeks, and for at least 4 years on storage at $-60{ }^{\circ} \mathrm{C}(215)$. Single freeze-thaw cycles are recommended as additional cycles result in a decrease in cotinine concentrations in serum (145).

Methods for the determination of nicotine concentrations in blood include RIA (140, 150, 151, 154), GC-NPD (253), GC-MS (126, 188), and HPLC-ECD (198). Cotinine concentrations in blood have been determined by RIA (17, 140, 145, 154, 164, 165), GC-NPD (4), GC-MS (189, 191, 192, 258), HPLC-UV (200, 164), and LC-API-MS-MS $(14,213-215)$. A number of methods have been established for the simultaneous determination of both nicotine and cotinine concentrations in blood by GC-FID $(124,125$, 127, 179, 181, 183-185), GC-MS (50, 128, 186, 193-195), HPLC-UV (164, 204), and LC-API-MS-MS (217, 219). trans-3'-Hydroxycotinine has been determined by GC-MS (186), and simultaneous analysis of nicotine, cotinine, trans-3'-hydroxycotinine, anabasine and nornicotine concentrations in blood by LC-MS-MS (219).

Cotinine concentrations in blood have been widely used to confirm self-reported smoking status $(4,7,9,17,153,192$, $194,236,243,249,258,259)$, and as a measure of smoke uptake by active smoking $(7,43,50,55,59,214,243,246$, $247,260,261)$. Cotinine concentrations in blood have also been extensively used to determine ETS exposure in adults $(14,16,17,214,262-266)$ and children (263-264, 267, 268). Cotinine concentrations in cord serum have been used as a measure of fetal exposure to cigarette smoke $(269,270)$.

The mean nicotine plasma elimination half-life of $2.3 \mathrm{~h}$ makes the determination of nicotine sensitive to short-term sampling variables and dependent on the time at which the last cigarette was smoked (71). As a consequence, most studies find only weak correlations between nicotine concentrations in blood and the nominal nicotine yield of cigarettes determined by machine smoking $(36,47,49,52,55)$, and between blood nicotine concentrations and the selfreported number of cigarettes smoked per day $(47,271)$. Nicotine concentrations in plasma are not significantly correlated with either cotinine or trans-3'-hydroxycotinine concentrations in plasma; however, cotinine and trans-3'hydroxycotinine concentrations in plasma are significantly correlated $(r=0.62, p<0.001)(87)$. Cotinine, trans-3hydroxycotinine, and trans-3'-hydroxycotinine glucuronide appear to be stable in plasma, but cotinine- $N$-glucuronide lacks stability (272). Cotinine concentrations in plasma are linearly and directly related to nicotine intake and show little variation for up to $2 \mathrm{~h}$ after the last expose to nicotine 
(273). Nicotine concentrations in plasma of non-smokers are also unrelated to ETS exposure (234). Nicotine concentrations in blood have, however, been extensively used as a biomarker to assess changes in smoking topography in experimental studies $(36,39,40,42,54,56,152,256$, 274-277) and in pharmacokinetic studies $(45,53,70,71$, $83,86,88,90,92,94,255)$.

The increase in the nicotine concentration in blood that occurs after smoking a single cigarette, sometimes referred to as "nicotine boost" (nicotine boost $_{\text {) }}$, is an individual measure of the absorbed dose of nicotine after smoking a single cigarette. Experimentally this is determined by measuring nicotine concentrations in blood prior to and after smoking a single cigarette and nicotine boost $_{\text {calculated as }}$ the difference between the post- and pre-smoking nicotine concentration in blood. As such, this is an experimental parameter and no standard protocol exists for its determination. Post-cigarette sampling of venous blood has been reported immediately after completion of the cigarette (256, 274, 277), 1 min (40, 42, 56, 152, 275, 276), 2 min $(37,42)$, within $3 \min (278), 3 \min (46), 5 \min (40,54), 10$ min $(151,275)$, and at various times (279) after taking the last puff of the cigarette. The time at which nicotine measurement is made after smoking the cigarette has a significant effect on the calculation of nicotine ${ }_{\text {boost }}$. HENNINGFIELD et al. (40) report a venous blood nicotine boost $_{\text {of }} 21.8$ $\pm 10.7 \mathrm{ng}$ nicotine $/ \mathrm{mL} 1 \mathrm{~min}$ after smoking a cigarette which decreases to $15.1 \pm 7.9 \mathrm{ng}$ nicotine $/ \mathrm{mL}$ at $5 \mathrm{~min}$. Nicotine $_{\text {boost }}$ is significantly associated with standard parameters of smoking topography (puff number, puff volume, and total puff volume) (46) and the number of puffs has the greatest effect on the blood nicotine increase (37, $42,275)$. A significant positive correlation is found between nicotine ${ }_{\text {boost }}$ and the nicotine yield of a cigarette (42, $56,152,275)$. Consistent with experimental studies on nicotine retention in the respiratory tract (34), a significant increase in the nicotine concentration in blood is not observed in the absence of inhalation (280), and the depth of inhalation has only a slight effect on nicotine ${ }_{\text {boost }}(275)$.

Representative data for cotinine concentrations in blood of smokers and nonsmokers are presented in Table 1. Cotinine concentrations in serum tend to show less circadian fluctuation than nicotine concentrations (255), and are an excellent biomarker for epidemiological studies, particularly if blood samples are drawn near to the time of the last exposure. Strong and significant correlations are found between the cotinine concentration in blood and the declared number of cigarettes smoked per day $(9,17,43,48,54,55,71,88,165$, $192,232,246,247,259,281)$, but not with the cigarette smoking machine yield of nicotine $(59,71)$. Cotinine concentration in serum measured after 8-10 h of smoking abstinence are linearly and directly related to daily intake of nicotine $(r=0.919 ; p<0.0001)$ (282). To differentiate smokers from non-smokers in population studies, serum cotinine cutoff values of 10.0-15.0 ng cotinine/mL serum have frequently been used as an indicator of active smoking $(14,17$, $214,246,247,249,258,261,264,283)$, and less than $0.1 \mathrm{ng}$ cotinine/mL serum to indicate absence of exposure to ETS in non-smokers (284). Although cut-off values of 10.0-15.0 ng cotinine/mL serum result in little overlap with the cotinine values usually encountered in active smokers, it has been suggested that a cut-off value of $15.0 \mathrm{ng}$ cotinine $/ \mathrm{mL}$ serum may be too high to accurately differentiate all smokers from non-smokers (249). Gender-specific cut-off values have also been suggested (285). Representative predictive power estimates for sensitivity (the true-positive fraction) and specificity (the false-positive fraction) of various cut-off values and self-reported smoking behaviour are presented in Table 2.

Various studies report that cotinine concentrations in serum are higher in African-Americans compared to other ethnic populations $(72,170,246,247,249,261-264,267)$. The higher cotinine concentrations in serum of AfricanAmericans remain after correction for the number of cigarettes smoked per day, nicotine content of the cigarettes smoked, and frequency of inhalation. African-Americans tend to smoke a significantly higher proportion of menthol cigarettes compared to Caucasians (286) and recent evidence suggests that smoking menthol cigarettes is associated with reduced nicotine C-oxidation and glucuronidation (99). Total cotinine concentrations in plasma, determined as the sum of cotinine, trans-3'-hydroxycotinine and their respective glucuronide conjugates (206) are not statistically different between African-Americans and Caucasians (272).

Cotinine concentrations in serum are significantly associated with self-reported exposure to $\operatorname{ETS}(14,17,262,281)$ and are about 2 times greater in subjects reporting exposure to ETS than in nonexposed subjects (281). Cotinine concentrations in serum are higher in non-smokers from smoking households compared to non-smokers from nonsmoking households $(14,264,266)$, and are significantly correlated with the number of smokers within the household (266). Cotinine concentrations in serum are also higher in African-American non-smokers compared to Caucasian non-smokers $(14,262)$ and other ethnic groups (14). Cotinine concentrations in plasma of adult non-smokers show a strong dose-response relationship with the smoking behaviour (reported as daily cigarette consumption) of either the spousal or co-habiting partner (265), and with hours/day of self-reported ETS exposure (14). Cotinine concentrations in serum are higher in children with smoking mothers compared to those with non-smoking mothers (263) and are higher in children from smoking households compared to non-smoking households (264, $267,268)$. Cotinine concentrations in serum from children are significantly associated with the number of cigarettes smoked within the home $(264,267,268)$ and are higher in African-American children compared to other ethnicities $(267,268)$. Total cotinine concentrations in plasma have also been investigated as a potential measure of nicotine exposure from ETS (287).

Maternal cotinine concentrations above $25 \mathrm{ng}$ cotinine $/ \mathrm{mL}$ serum are invariably associated with detectable concentrations in fetal compartments, and mean cotinine concentrations are higher in fetal compared to maternal serum (162). Nicotine readily enters the fetal compartment via the placenta and fetal concentrations are generally higher than in the mother. At term, cotinine concentrations in cord blood serum correlate well with the average number of cigarettes smoked per day $(r=0.46 ; p=0.003)$ and with thiocyanate concentrations in cord blood $(r=0.63 ; p$ $<0.001)$ (166). Cotinine concentrations in maternal and fetal serum are significantly correlated during the first half 


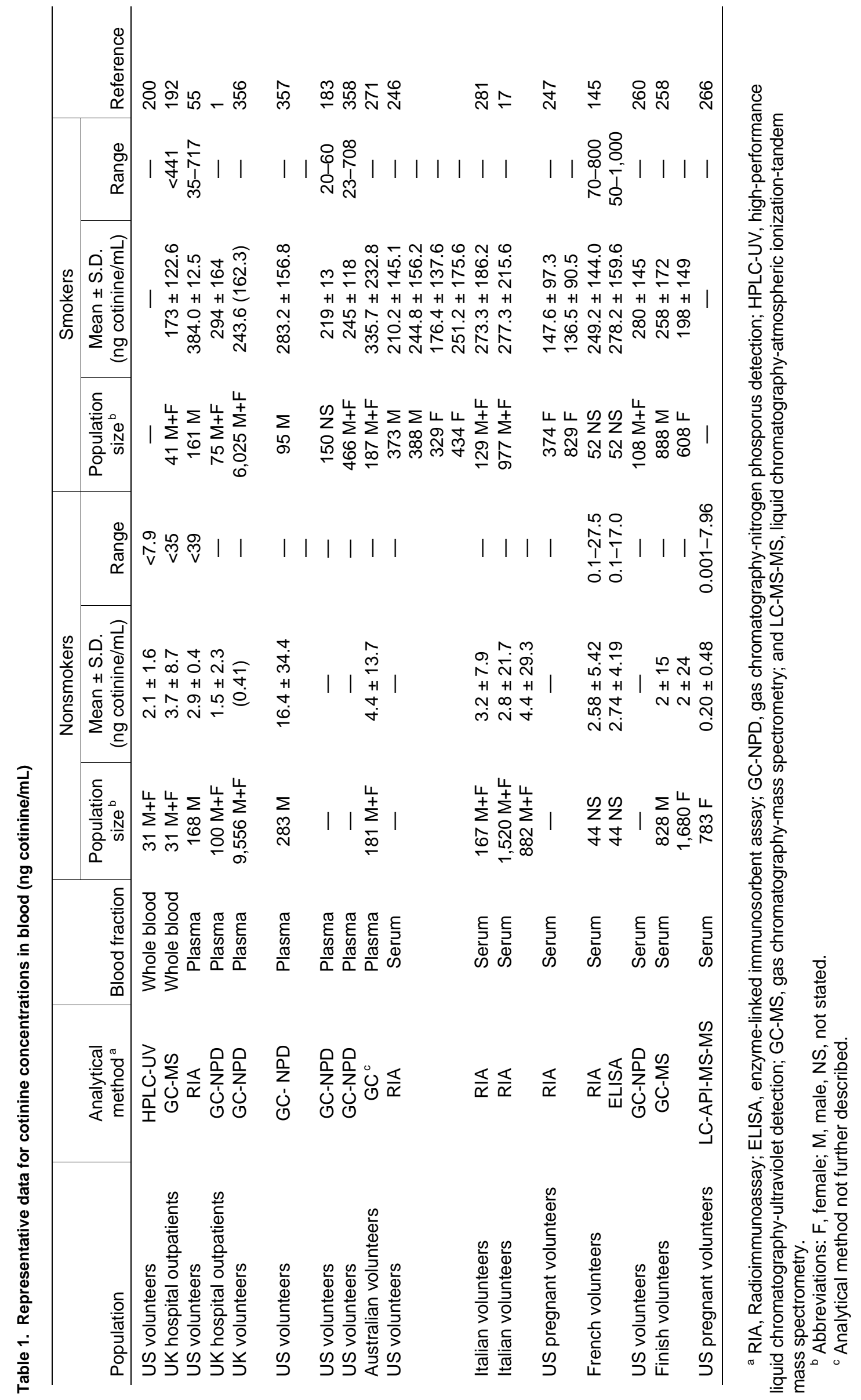


Table 2. Representative blood serum cotinine cut-off values and predictive power (sensitivity and selectivity) for the differentiation of smokers and nonsmokers

\begin{tabular}{|c|c|c|c|c|c|c|}
\hline Population & $\begin{array}{l}\text { Analytical } \\
\text { method }^{a}\end{array}$ & $\begin{array}{l}\text { Population } \\
\text { size }^{\text {b }}\end{array}$ & $\begin{array}{l}\text { Cotinine cut-off } \\
\text { (ng/mL serum) }\end{array}$ & $\begin{array}{c}\text { Sensitivity } \\
(\%)\end{array}$ & $\begin{array}{c}\text { Specificity } \\
(\%)\end{array}$ & Reference \\
\hline US volunteers & RIA & $5,115 \mathrm{M}+\mathrm{F}$ & $\begin{array}{c}19 \\
17 \\
15 \\
14 \\
13 \\
11 \\
9 \\
7 \\
5\end{array}$ & $\begin{array}{l}93.2 \\
93.9 \\
94.5 \\
94.9 \\
95.0 \\
95.4 \\
96.0 \\
96.4 \\
97.1\end{array}$ & $\begin{array}{l}96.5 \\
96.3 \\
96.0 \\
95.8 \\
95.6 \\
95.1 \\
94.6 \\
93.1 \\
89.8\end{array}$ & 243 \\
\hline Italian volunteers & RIA & $3,379 \mathrm{M}+\mathrm{F}$ & 15 & 94.8 & 95.6 & 17 \\
\hline US pregnant volunteers & RIA & $3,343 \mathrm{~F}$ & 14 & 93.5 & 98.0 & 247 \\
\hline US volunteers & RIA & $5,115 \mathrm{M}+\mathrm{F}$ & $\begin{array}{l}13 \\
13\end{array}$ & $\begin{array}{l}96.6 \\
92.9\end{array}$ & $\begin{array}{l}96.5 \\
93.5\end{array}$ & 264 \\
\hline Australian volunteers & $\mathrm{GC}^{\mathrm{c}}$ & $368 \mathrm{M}+\mathrm{F}$ & 44 & 95.2 & 98.3 & 271 \\
\hline Swedish volunteers & GC-NPD & $496 \mathrm{~F}$ & 17.5 & 100.0 & 93.9 & 4 \\
\hline US volunteers & GC-NPD & $736 \mathrm{M}+\mathrm{F}$ & 14 & 87.9 & 93.7 & 248 \\
\hline Finish volunteers & GC-MS & $5,846 \mathrm{M}+\mathrm{F}$ & $\begin{array}{l}10 \mathrm{M} \\
10 \mathrm{~F}\end{array}$ & $\begin{array}{l}97.2 \\
94.7\end{array}$ & $\begin{array}{l}93.7 \\
54.8\end{array}$ & 258 \\
\hline US volunteers & LC-MS-MS & $15,357 \mathrm{M}+\mathrm{F}$ & 15 & 92.5 & 98.6 & 249 \\
\hline US volunteers & LC-MS-MS & $2,107 \mathrm{M}+\mathrm{F}$ & $\begin{array}{c}15 \\
11.1 \\
10\end{array}$ & $\begin{array}{l}75.0 \\
78.9 \\
79.4\end{array}$ & $\begin{array}{l}97.8 \\
97.3 \\
96.7\end{array}$ & 244 \\
\hline
\end{tabular}

${ }^{a}$ RIA, Radioimmunoassay; GC-NPD, gas chromatography-nitrogen phosporus detection; GC-MS, gas chromatography-mass spectrometry; and LC-MS-MS, liquid chromatography-tandem mass spectrometry.

${ }^{\mathrm{b}}$ Abbreviations: $\mathrm{F}$, female; M, male, NS, not stated.

${ }^{\mathrm{c}}$ Analytical method not further described.

of pregnancy $(r=0.97 ; p<0.001)(162)$ and at term $(269$, $270,288)$. At term, cotinine concentrations in cord blood serum are significantly associated with self-reported maternal cigarette smoking and with self-reported exposure to ETS (270). Linear regression analysis suggests that serum concentrations in cord blood increase by $4.4 \mathrm{ng}$ cotinine/mL for each cigarette smoked per day (166). Cotinine concentrations of $14 \mathrm{ng}$ cotinine/mL cord blood serum are reported to distinguish between active maternal smoking and non-smoking (166). Lower cut-off values of $1.0 \mathrm{ng}$ cotinine $/ \mathrm{mL}$ cord blood serum (289) and $1.78 \mathrm{ng}$ cotinine/mL cord blood serum (270) have been suggested to discriminate newborns from non-smokers with and without exposure to ETS.

Daily nicotine intake $\left(D_{\text {nic }}\right)$ can be estimated based on the fractional conversion of nicotine to cotinine, and the clearance of cotinine for an individual (71):

$$
D_{\text {nic }}(\mathrm{mg} / 24 \mathrm{~h})=K \times \text { Plasma }_{\text {cotinine }}(\mathrm{ng} / \mathrm{mL})
$$

On average, $K=0.08$ with a range of 0.05 to 0.10 (coefficient of variation, $21.9 \%$ ). In adult smokers, cotinine concentrations in blood of $12.5 \mathrm{ng}$ cotinine $/ \mathrm{mL}$ equates to a daily nicotine intake of approximately $1 \mathrm{mg}$ (13). The $K$ factor and cotinine concentrations in plasma are suggested to provide an estimate of daily nicotine intake from both active smoking and exposure to ETS in non-smokers (290).

\section{Saliva}

For basic drugs with a $\mathrm{pKa}>6.0$ such as nicotine $(\mathrm{pKa}=$ 3.2 and 7.9) (255), saliva concentrations are poorly correlated with free plasma drug concentrations since secretion into saliva is highly dependent on salivary $\mathrm{pH}$. For coti- nine, which is a weaker base than nicotine, saliva concentrations are less $\mathrm{pH}$ dependent and correlate well with the free drug concentrations in blood $(89,214,233,235)$. Standardization of saliva collection techniques is essential for saliva cotinine measurements. Saliva specimins are likely to be affected by both time from last meal and by time since smoking the last cigarette.

Nicotine concentrations in saliva are seldom measured due to the poor correlation between saliva nicotine and the number of cigarettes smoked per day, and between saliva nicotine and the nicotine yield per cigarette (291). In addition, direct contact of saliva with nicotine present in tobacco smoke during smoking results in artificially high concentrations of nicotine in saliva which do not reflect the blood nicotine concentration. Nicotine concentrations in saliva show a linear increase with self-reported exposure to ETS although this biomarker is only sensitive to very recent exposure to ETS (234).

Cotinine concentrations in saliva have been extensively determined as a biomarker of smoke exposure in field studies since collection of saliva is non-invasive and easy to perform $(1,7,292,293)$. Representative data are presented in Table 3. Saliva sample collection is ideal for use with children or when multiple sample collections are required over time. Samples can also be collected by the study subjects in the absence of trained personnel and returned by mail for subsequent analysis (257, 294, 295). Cotinine concentrations in saliva are stable even when samples remain unfrozen for 12 days and are returned by mail (257). Similarly, cotinine concentartions in saliva stored on Salivette swabs remain stable at room temperature for 14 days (214). However, collection in the absence of trained personnel may compromise compliance to the study protocol and integrity of the sample. This may be a 
Table 3. Representative data for cotinine concentrations in saliva (ng cotinine/mL saliva)

\begin{tabular}{|c|c|c|c|c|c|c|c|c|}
\hline \multirow[b]{2}{*}{ Population } & \multirow[b]{2}{*}{$\begin{array}{c}\text { Analytical } \\
\text { method }^{\mathrm{a}}\end{array}$} & \multicolumn{3}{|c|}{ Nonsmokers } & \multicolumn{3}{|c|}{ Smokers } & \multirow[b]{2}{*}{ Reference } \\
\hline & & $\begin{array}{l}\text { Population } \\
\text { size }\end{array}$ & $\begin{array}{c}\text { Mean } \pm \text { S.D. } \\
\text { (ng cotinine } / \mathrm{mL})\end{array}$ & Range & $\begin{array}{l}\text { Population } \\
\text { size }\end{array}$ & $\begin{array}{c}\text { Mean } \pm \text { S.D. } \\
\text { (ng cotinine } / \mathrm{mL})\end{array}$ & Range & \\
\hline US volunteers & RIA & - & - & - & $\begin{array}{l}108 \mathrm{M} \\
186 \mathrm{~F}\end{array}$ & $\begin{array}{l}350.3 \pm 206.6 \\
267.8 \pm 191.9\end{array}$ & - & 60 \\
\hline Canadian volunteers & RIA & $\begin{array}{l}125 \mathrm{M} \\
123 \mathrm{~F}\end{array}$ & $\begin{array}{l}1.1 \pm 1.6 \\
1.5 \pm 2.3\end{array}$ & $\begin{array}{l}0.1-13.3 \\
0.1-14.7\end{array}$ & - & - & - & 298 \\
\hline US volunteers & RIA & $30 \mathrm{M}+\mathrm{F}$ & $0.3 \pm 1.6$ & $1-9$ & $60 \mathrm{M}+\mathrm{F}$ & $349.2 \pm 195.4$ & $26-933$ & 292 \\
\hline US pregnant volunteers & RIA & $476 \mathrm{~F}$ & $1.1 \pm 5$ & - & $\begin{array}{l}144 \mathrm{~F} \\
323 \mathrm{~F} \\
258 \mathrm{~F}\end{array}$ & $\begin{array}{l}198.7 \pm 120 \\
106.4 \pm 68 \\
126.8 \pm 94\end{array}$ & - & 359 \\
\hline UK volunteers & GC-NPD & $355 \mathrm{~F}$ & $4.6 \pm 6.3$ & $<83$ & $173 \mathrm{~F}$ & $123.3 \pm 133.8$ & $<906$ & 245 \\
\hline Swiss volunteers & GC-NPD & $97 \mathrm{M}+\mathrm{F}$ & $6.3 \pm 24.2$ & $<46$ & $207 \mathrm{M}+\mathrm{F}$ & $166 \pm 170$ & $<838$ & 295 \\
\hline UK hospital outpatients & GC-NPD & $100 \mathrm{M}+\mathrm{F}$ & $1.7 \pm 2.3$ & - & $75 \mathrm{M}+\mathrm{F}$ & $330 \pm 190$ & - & 1 \\
\hline UK volunteers & GC-NPD & $292 M+F$ & 1.74 & - & $275 M+F$ & 295.2 & - & 232 \\
\hline UK volunteers & GC-NPD & $245 \mathrm{~F}$ & $0.48 \pm 0.74$ & $<4.5$ & $170 \mathrm{~F}$ & $146.2 \pm 92.8$ & $<480$ & 250 \\
\hline Southeast Asian volunteers & HPLC-UV & - & - & - & $327 M+F$ & $65 \pm 43$ & - & 233 \\
\hline US volunteers & Not stated & - & - & - & $228 M+F$ & $286.8 \pm 178.7$ & $<967$ & 238 \\
\hline
\end{tabular}

${ }^{a}$ RIA, Radioimmunoassay; ELISA, enzyme-linked immunosorbent assay; GC-NPD, gas chromatography-nitrogen phosporus detection; and HPLC-UV, high-performance liquid chromatography-ultraviolet detection.

Table 4. Representative saliva cotinine cut-off values and predictive power (sensitivity and selectivity) for the differentiation of smokers and nonsmokers

\begin{tabular}{|c|c|c|c|c|c|c|}
\hline Population & $\begin{array}{l}\text { Analytical } \\
\text { method }^{a}\end{array}$ & Population size ${ }^{b}$ & $\begin{array}{l}\text { Cotinine cut-off } \\
\text { (ng/mL saliva) }\end{array}$ & Sensitivity (\%) & Specificity (\%) & Reference \\
\hline US pregnant volunteers & RIA & $814 \mathrm{~F}$ & 30 & 86.2 & 73.8 & 3 \\
\hline \multirow[t]{5}{*}{ US volunteers } & RIA & $5,887 \mathrm{M}+\mathrm{F}$ & 19 & 99.1 & 89.8 & 10 \\
\hline & & & 19 & 99.2 & 91.7 & \\
\hline & & & 19 & 98.2 & 91.5 & \\
\hline & & & 19 & 98.4 & 88.2 & \\
\hline & & & 19 & 95.6 & 91.7 & \\
\hline \multirow[t]{3}{*}{ US volunteers } & RIA & $1,881 \mathrm{M}+\mathrm{F}$ & 10 & 87.2 & 97.6 & 239 \\
\hline & & & 10 & 81.1 & 98.1 & \\
\hline & & & 10 & 62.2 & 98.2 & \\
\hline UK volunteers & GC-NPD & $579 \mathrm{~F}$ & 14 & 95 & 96 & 250 \\
\hline UK volunteers & GC-NPD & $211 \mathrm{M}+\mathrm{F}$ & 14.2 & 96 & 99 & 1 \\
\hline UK volunteers & GC-NPD & $508 \mathrm{~F}$ & 14.7 & 100 & 98.5 & 245 \\
\hline \multirow[t]{2}{*}{ Swiss volunteers } & GC-NPD & $319 \mathrm{M}+\mathrm{F}$ & 13 & 86.5 & 95.9 & 295 \\
\hline & & & 7 & 92.3 & 89.7 & \\
\hline US volunteers & GC-NPD & $236 \mathrm{M}+\mathrm{F}$ & 10 & 99.1 & 100.0 & 240 \\
\hline
\end{tabular}

${ }^{a}$ RIA, Radioimmunoassay; and GC-NPD, gas chromatography-nitrogen phosporus detection.

${ }^{\mathrm{b}}$ Abbreviations: $\mathrm{F}$, female; $\mathrm{M}$, male.

concern in epidemiological studies in which cotinine concentrations in saliva are used to estimate nicotine concentrations in blood. Cotinine concentrations in saliva are dependent on the method of sample collection and are lower in stimulated compared to unstimulated saliva (296). Saliva cotinine has a half-life of about $17.5 \mathrm{~h}$ and shows some diurnal variation, therefore, a single spot saliva sample may not fully represent steady-state conditions (71).

Nicotine concentrations in saliva have been determination by ELISA (143), RIA (159), and GC with nitrogen-sensitive detection $(250,253)$. Cotinine has been determined by ELISA (143-145), RIA (154), HPLC-UV (200, 233), GCMS (191), and LC-API-MS-MS (212, 214). Simultaneous analysis of nicotine and cotinine concentrations in saliva is possible by GC with nitrogen-sensitive detection (127, 297), GC-MS (194, 196), and LC-MS-MS (213). Cotinine and trans-3'-hydroxycotinine concentrations in saliva have been determined by LC-API-MS-MS (212). Cotinine concentrations in saliva have been widely used to confirm self-reported smoking status $(1,3,7,10,236,239,246,250,293)$, as a measure of smoke uptake by active smoking $(60,63,64,232$, $233,238,295)$, and to determine ETS exposure in adults (88, 214, 265, 293, 298) and children (144, 297, 299).

Cotinine concentrations in saliva are usually $10-40 \%$ higher than cotinine concentrations in plasma of the same individual $(8,83,84,200,214,230-232)$. This ratio is found at all levels of smoking activity and exposure to ETS in non-smokers, and at a steady-state concentration of cotinine after intravenous infusion of nicotine (83). Cotinine concentrations in saliva can be used with reasonable accuracy (approximately $\pm 10 \%$ ) to predict both cotinine concentrations in plasma (194) and serum (214). The saliva:plasma ratio is higher among nonsmokers compared to smokers (214). The ratio is somewhat lower in younger people than in older people, and is significantly affected by body mass index. Higher body mass index is associated with lower salivary cotinine concentrations (64). To discriminate between smokers and non-smokers, most studies have used cut-off values ranging between $7 \mathrm{ng}$ cotinine/mL (295) and $44 \mathrm{ng}$ cotinine/mL saliva (300) as shown in Table 4. However, cut-off values as high as $100 \mathrm{ng}$ coti- 
nine/mL saliva have also been used (301). One study has assessed self-reported smoking status and biochemical verification using salivary cotinine at baseline and at five annual follow-up visits (10). Compared to self-report, sensitivity and specificity of cotinine was stable over five years. Similar to cotinine concentrations in serum $(72,170,246,247,249$, 261-264, 267), ethnic differences in cotinine concentrations in saliva have been found suggesting that different cut-off values may be required to determine smoking status in African-Americans compared to Caucasians (3, 238, 243). Maximum sensitivity and specificity is reported with a cutoff value of $25 \mathrm{ng}$ cotinine/mL saliva for African-Americans and $11 \mathrm{ng}$ cotinine/mL saliva for Caucasians (3). Slightly lower cut-off values of $16 \mathrm{ng}$ cotinine/mL for African-Americans and $9 \mathrm{ng}$ cotinine/mL for Caucasians have also been reported (243). A cut-off value of $15 \mathrm{ng}$ cotinine/mL saliva is commonly used to differentiate smokers from non-smokers $(214,242,245,283)$. It has been recommended that $\leq 30$ ng cotinine/mL saliva should be considered to be the upper cut-off value for discriminating non-smokers exposed to ETS from active smokers $(293,301)$.

Cotinine concentrations in saliva are only weakly associated with the nominal nicotine yield of cigarettes $(60,64,295)$, but show a significant correlation with the estimated total nicotine absorption per day (63). Strong correlations are found between the number of self-reported cigarettes smoked per day and cotinine concentrations in saliva $(r=$ 0.53; $p<0.0001)$ and $\mathrm{CO}$ in exhaled breath $(r=0.52 ; p<$ $0.0001)(60)$. Other studies confirm a significant correlation between the number of self-reported cigarettes smoked per day and cotinine concentrations in saliva $(3,232,250,294)$. Most studies suggest that non-smokers typically have cotinine concentrations in saliva less than $5.0 \mathrm{ng} / \mathrm{mL}$ (301). Adult self-reports of exposure to ETS are not strongly associated with the cotinine concentration in saliva, and regression models can seldom account for more than $20 \%$ of the variance in $\log$ cotinine concentrations $(237,298,302)$. Cotinine concentrations in saliva are significantly higher in children from smoking households compared to non-smoking households (144, 297, 299). Self-reported maternal smoking rather than paternal smoking results in higher cotinine concentrations in childrens saliva $(297,299)$. The highest cotinine concentrations in saliva are found in children with two smoking parents (297).

\section{Urine}

Urinary excretion of nicotine is strongly influenced by both urine $\mathrm{pH}$ and rate of urine flow $(253,303)$. It can be assumed that a smoker reaches a steady state concentration of nicotine, which implies that the daily amount of nicotine uptake is equal to the amount of nicotine and metabolites excreted in 24-h urine. Although nicotine concentrations in urine correlate fairly well with nicotine intake (121), the short half-life of nicotine $\left(t_{1 / 2} \sim 2.6 \mathrm{~h}\right)(90)$ precludes its use as an accurate marker for assessing tobacco use in smokers that occurred more than about 10 hours previously. Similarly, excretion of nicotine in urine cannot be used to accurately discriminate non-smokers exposed to ETS from active smokers.

The collection of 24-h urine samples is often impractical and spot urine samples tend to be more commonly used.
For practical reasons, measurement of creatinine concentration in urine is commonly used as a correction factor to compensate for differences in renal function and urinary dilution, and urinary concentrations expressed as a ratio to urinary creatinine $(12,24,161,177,236,304-308)$. However, some evidence suggests that a simple ratio is insufficient and regression adjustment is required (305). The use of urine specific gravity has also been suggested as a reliable means of adjusting cotinine concentrations in urine for sample dilution (309) and techniques for normalization of urine concentrations using creatinine or specific gravity reported (310). Whether a correction factor for diuresis is actually required has been challenged $(283,311)$. Collection of first-day urine from young infants is possible using either adhesive collection bags $(270,312)$ or from diapers (304). Appropriate storage of collected samples is required (frozen at $-20{ }^{\circ} \mathrm{C}$ ) since storage of urine samples at elevated temperatures over long periods may result in thermal degradation of cotinine- $N$-glucuronide and a resultant increase in cotinine concentrations in urine (313). Under appropriate storage conditions (frozen urine samples at $\left.-20{ }^{\circ} \mathrm{C}\right), 10$ year stability of cotinine has been determined (314). However, even under these conditions the stability of trans-3'-hydroxycotinine is poor (219).

Nicotine concentrations in urine have been determined by RIA $(140,154,315)$ and by GC with a nitrogen-sensitive detector (253). Methods for the determination of cotinine concentrations in urine include EIA (148), ELISA (144), FIA (146), RIA (12, 140, 154, 161, 165, 315), GC-MS (148, 161, 189-191), HPLC-UV $(58,202)$, and test-strip assays (223-225). Several methods allow the joint determination of nicotine and cotinine concentrations in urine by GC-FID (184), GC with a nitrogen-sensitive detector $(127,298)$, and GC-MS (193, 194, 196). trans-3'-Hydroxycotinine in urine has been determined by $\mathrm{GC}$ with either a nitrogen-sensitive detector or by GC-MS (182), and nicotine, cotinine and trans-3'-hydroxycotinine by GC with a nitrogen-sensitive detector (181), GC-MS (186), and LC-MS-MS (211). Multiple nicotine metabolites have been determined by GC-thermionic-specific detector (62), GC-MS (21), thermospray LC-MS $(63,74,210,211)$, and LC-MS-MS $(98,222)$.

Cotinine concentrations in urine have been widely used to confirm self-reported smoking status $(1,58,161,202,236$, $314,316)$, and to monitor smoking cessation $(24,26)$. Similarly, cotinine concentrations in urine have also been used to determine ETS exposure in adults $(12,302,314$, $316)$ and children $(170,308,317,318)$, and to measure fetal exposure to cigarette smoke $(306,307,315,319)$. Excretion of cotinine in urine accounts for about $10 \%$ of the total dose of systemic nicotine (Figure 1), therefore, even a small perturbation in the metabolism of nicotine to cotinine could have a large effect on predicting total systemic nicotine exposure using cotinine as a biomarker (76). It is difficult to define a cut-off limit for differentiation of smokers from non-smokers in spot urine samples although a value of $50 \mathrm{ng}$ cotinine/mL urine has been recommended (283). After correction for creatinine excretion, suggested cut-off values range from $28 \mathrm{ng}$ cotinine/mg creatinine (202) to $550 \mathrm{ng}$ cotinine/mg creatinine (26). However, a cut-off value of $150 \mathrm{ng}$ cotinine/mg creatinine appears to be the most appropriate (316). A reference range of 1.1-90.0 $\mu \mathrm{mol}$ cotinine/mol creatinine for non-smokers 
Table 5. Reported mean urinary excretion as a molar percentage ( $\% \pm$ SD) of total recovered nicotine and metabolites in smokers urine $^{\text {a }}$

\begin{tabular}{lc|c|c|c|c|c|c}
\hline & \multicolumn{7}{c}{ Study (reference) } \\
& \multicolumn{7}{c}{ Number of subjects } \\
\cline { 2 - 8 } & $1(62)$ & $2(74)$ & $3(93)$ & $4(97)$ & $5(98)$ & $6(319)$ & $7(319)$ \\
\hline Cotinine & 91 & 11 & 12 & 4 & 5 & 166 \\
Nicotine & $9.2 \pm 2.6$ & $13.2 \pm 3.9$ & $13.3 \pm 3.1$ & $14.8 \pm 5.9$ & 15.2 & $10.7 \pm 2.4$ & $11.1 \pm 3.2$ \\
trans-3'-Hydroxycotinine & $9.4 \pm 5.7$ & $10.4 \pm 3.7$ & $10.4 \pm 4.4$ & $7.9 \pm 4.6$ & 9.5 & $11.2 \pm 5.0$ & $8.8 \pm 5.6$ \\
Cotinine- $N$-glucuronide & $36.1 \pm 10.6$ & $35.2 \pm 7.4$ & $39.1 \pm 12.5$ & $42.4 \pm 12.8$ & 34.1 & $31.5 \pm 8.8$ & $31.4 \pm 9.0$ \\
Nicotine- $N$-glucuronide & $14.0 \pm 5.4$ & $17.5 \pm 6.3$ & $15.8 \pm 7.8$ & $12.1 \pm 6.0$ & 20.1 & $13.4 \pm 5.2$ & $14.8 \pm 5.5$ \\
trans-3'-Hydroxycotinine-O- & $4.5 \pm 2.5$ & $2.8 \pm 2.2$ & $4.6 \pm 2.9$ & $2.6 \pm 2.1$ & 3.7 & $5.1 \pm 2.4$ & $5.4 \pm 2.9$ \\
$\quad$ glucuronide & $22.8 \pm 10.0$ & $8.5 \pm 3.8$ & $7.8 \pm 5.9$ & $10.3 \pm 7.6$ & 7.4 & $6.3 \pm 3.3$ & $6.8 \pm 5.1$ \\
Nicotine $N$-1'-oxide & $3.0 \pm 2.1$ & $6.8 \pm 2.9$ & $3.7 \pm 0.9$ & N.D. & 6.7 & $4.2 \pm 1.8$ & $4.5 \pm 2.0$ \\
Cotinine $N$-1-oxide & $0.9 \pm 0.9$ & $3.9 \pm 1.9$ & $4.5 \pm 1.5$ & N.D. & 2.2 & N.D. & N.D. \\
Nornicotine & - & - & $0.6 \pm 0.2$ & - & - & $0.5 \pm 0.3$ & $0.7 \pm 0.2$ \\
Norcotinine & N.D. & $1.5 \pm 0.5$ & N.D. ${ }^{\mathrm{a}}$ & N.D. & 1.3 & $2.1 \pm 0.4$ & $2.2 \pm 0.5$ \\
Percentage sum & 99.8 & 99.8 & $99.9^{\mathrm{b}}$ & 90.1 & 100.2 & $97.9^{\mathrm{c}}$ & $97.1^{\mathrm{c}}$ \\
\hline
\end{tabular}

${ }^{a}$ N.D., not determined.

${ }^{\mathrm{b}}$ Percentage sum included 4-hydroxy-4-(3-pyridyl)butanoic acid and 4-oxo-4-(3-pyridyl)butanoic acid.

${ }^{c}$ Percentage sum includes 5'-hydroxycotinine, 4-hydroxy-4-(3-pyridyl)butanoic acid, 4-oxo-4-(3-pyridyl)butanoic acid, and 3-pyridylacetic acid.

have been established according to International Union of Pure and Applied Chemistry (IUPAC) and International Federation for Clinical Chemistry (IFCC) recommended statistical methods for use in risk assessment of exposure to tobacco smoke (177). trans-3'-Hydroxycotinine, the predominant metabolite of nicotine in urine, has also been suggested as a better indicator of smoking status (21). However, the use of this biomarker for this purpose has seldom been reported.

Cotinine concentrations in urine correlate modestly with the number of self-reported cigarettes smoked per day $(24,162$, $165,320,321)$. Significant correlations have been found after correction for urinary creatinine $(r=0.442 ; p<$ 0.0001 ), and each cigarette smoked is associated with an increase in cotinine excretion of $41 \mathrm{ng}$ cotinine/mg creatinine (161). Small but significant correlations are observed between the sum of nicotine, cotinine and trans-3'-hydroxycotinine expressed as $\mathrm{ng} / \mathrm{mg}$ creatinine in spot urine samples and cigarette nicotine yield $(r=0.23 ; p<0.001)$ and number of self-reported cigarettes smoked per day $(r=0.46 ; p<$ 0.001) (65). Cotinine concentrations in serum and urine are highly correlated in smokers in the absence of correction for urinary creatinine $(305,311)$ and after correction for urinary creatinine $(r=0.91)(305)$.

The determination of nicotine plus seven main metabolites in urine (i.e., nicotine, cotinine, trans-3'-hydroxycotinine and their corresponding glucuronic acid conjugates, nicotine- $N$-1'-oxide and cotinine- $N$-1-oxide) accounts for approximately $90 \%$ to $95 \%$ of the administered intravenous dose of nicotine in tobacco users (62). This is in good agreement with a second mass balance study in which the determination of nicotine plus eight major metabolites in urine (nicotine, cotinine, trans-3'-hydroxycotinine and their corresponding glucuronic acid conjugates, nicotine$N$-1'-oxide, cotinine- $N$-1-oxide, and nornicotine) accounted for approximately $88 \%$ of the systemic dose of nicotine, with similar patterns of metabolites in smokers and subjects receiving transdermal nicotine (93). However, large interindividual variability in the percentage excretion of individual metabolites is evident, and the excretion profile of individual nicotine metabolites in urine is significantly different during pregnancy compared to postpartum (95). In theory, the best estimate of nicotine intake would be obtained by measuring the amount of nicotine and all of its metabolites in 24-h urine samples, i.e., the determination of "total nicotine equivalents" in urine $(93,322,323)$. However, the determination of multiple metabolites is complex (74) and few studies have used this approach to assess nicotine disposition in smokers (Table 5). Determination of nicotine, cotinine, trans-3'-hydroxycotinine, and their glucuronide conjugates accounts for approximately $80 \%$ ot total nicotine excretion in urine (323, and Table 5).

Total nicotine uptake and excretion can be determined by either indirect analysis of nicotine-derived glucuronides via quantitation of the aglycone before and after hydrolysis with $\beta$-glucuronidase $(21,44,62,63,73,74,86,88,93$, $95,97)$ or by direct analysis of the glucuronide conjugates (98). A similar approach has been used to estimate nicotine exposure from transdermal nicotine patches by determination of total cotinine (cotinine plus its glucuronide conjugate) and total trans-3'-hydroxycotinine (trans-3'-hydroxycotinine and its glucuronide conjugate) in urine (21).

Cotinine concentrations in urine show a positive and significant correlation with self-reported exposure to ETS in adults $(12,234,302)$. Cotinine concentrations in urine are higher in adults living in a smoking household compared to adults living in a non-smoking household $(12,302)$. Cotinine concentrations are approximately three times higher in nonsmokers with a spouse or partner who smokes (mean, 11.4 ng cotinine/mL urine) compared to a spouse or partner who is a non-smoker (mean, $4.4 \mathrm{ng}$ cotinine/mL urine) (321). Consistent with this, cotinine concentrations in urine are significantly higher in children from smoking households compared to non-smoking households $(144,317,318)$, and self-reported maternal smoking in the home is significantly correlated with the cotinine concentrations in children's urine $(170,308)$. The cumulative number of family members who smoke correlates with cotinine concentration in children's urine, and maternal smoking is the single most important contributor (170). 
Statistically significant associations are found between nicotine and cotinine concentrations in maternal urine samples collected prior to birth and urine samples from newborns collected after birth $(r=0.897 ; p>0.001$ and $r=$ $0.921 ; p<0.001$, respectively) (307). When nicotine and cotinine concentrations in urine are adjusted for creatinine, the significance of both associations are no longer evident ( $r=0.318 ; p=0.14$ and $r=0.098 ; p=0.68$, respectively). In contrast, urinary trans-3'-hydroxycotinine excretion in maternal and newborn urine is significantly associated both before $(r=0.825 ; p<0.001)$ and after correction for creatinine $(r=0.871 ; p<0.001)$. However, these associations are only found in active smokers and are difficult to interpret using spontaneous collection of urine samples.

Cotinine concentrations in urine after correction for creatinine are higher in breast-fed babies from smoking mothers $(<3,519 \mathrm{ng}$ cotinine/mg creatinine $)$ compared to bottlefed babies $(<1,458 \mathrm{ng}$ cotinine/mg creatinine) $(304,319$, $324)$, and the cotinine concentration in urine of breast-fed babies is associated with maternal cigarette consumption in the previous $24 \mathrm{~h}(304,320,325)$. However, the ranges of cotinine concentrations in urine for any given number of cigarettes smoked are wide and the associations, when measured as correlation coefficients, are weak.

\section{Hair}

Hair is widely used in forensic science and toxicology to determine drug exposure over a prolonged period (326, 327 ), and several studies have investigated the potential use of nicotine and cotinine incorporation in scalp hair as long-term biomarkers of nicotine exposure (reviewed in 328). Although hair samples are indefinitely stable and can be easily collected (327), analysis of hair samples suffers from several drawbacks. Difficulties can be encountered in extraction of nicotine and cotinine in a reproducible manner, the effect of hair treatment on nicotine and cotinine concentrations is mainly unknown, and there are no reference materials with known quantities of both analytes to assess and compare various methodologies (329). Measuring nicotine and cotinine concentrations in hair samples from infants may not be feasible in many infants with minimal hair growth, and there may be restrictions on cutting hair in some cultures.

Nicotine in hair is mainly incorporated through systemic absorption in the hair follicle resulting in a constant axial concentration of nicotine along the hair shaft $(330,331)$, and to a minor extent by passive absorption from the atmosphere (332). Atmospheric absorption of nicotine results in higher nicotine concentrations in the distal part of the hair shaft compared to proximal hair sampled close to the scalp (157, 333 ). In contrast, cotinine is primarily incorporated into hair by systemic absorption from the hair follicle (334). trans-3'Hydroxycotinine has not been reported in hair. The chemical mechanism of drug accumulation and retention in hair has not been fully elucidated, but hair pigmentation and melanin content appears to be an important factor determining the extent of nicotine incorporation $(157,331,335)$. In vitro studies suggest that both nicotine and cotinine form adducts via the interaction of the 4-pyridinyl radical with dopaquinone, a precursor intermediate in the biosynthesis of melanin (336). Hair treatment, in particular the use of dyes, permanent wave, and hydrogen peroxide which may damage the structure and integrity of hair, reduce both nicotine and cotinine concentrations (329).

Both nicotine and cotinine concentrations in hair are sensitive to differences in analytical methods, in particular, sample washing and extraction $(157,199,329)$. A range of solvents have been used to wash hair to remove passive absorption of nicotine, these include ethanol, methanol, dichloromethane, and detergents $(329,330,337)$; however, it appears that dichloromethane is the most effective (329). Following neutralization with concentrated hydrochloric acid, nicotine and cotinine have mainly been determined by RIA (157-159, 315), GC-NPD (331, 335), GC-MS (196, 326, 330,332 ), and HPLC-UV detection at $254 \mathrm{~nm}$ (329).

Measurement of nicotine and cotinine concentrations in hair has been used to determine smoking status $(157,330)$, changes in smoking behaviour in smoking cessation programmes $(331,338)$, exposure to ETS $(159,170,199,334)$, and to determine intrauterine exposure of newborns to nicotine from maternal smoking during pregnancy $(158,315$, 337, 339).

Some controversy surrounds the reported association between nicotine and cotinine concentrations in hair with the number of self-reported cigarettes smoked. Several studies show no correlation between nicotine and cotinine concentrations in hair with self-reported smoking (158, 339), while other studies report a significant correlation between the number of self-reported cigarettes smoked per day and hair nicotine $(315,331,337)$ and hair cotinine $(315,337)$. The lack of an association observed in some studies may be due to smoking deception in women smokers (243) and under-reporting of the amount smoked in studies investigating maternal smoking during pregnancy (340). A cutoff value of $>2$ ng nicotine/mg hair has been found to differentiate between smokers and non-smokers (341). In neonatal hair, a correlation with maternal smoking has been observed for cotinine, but not for nicotine concentrations in hair (315).

Strong correlations have been found between reported exposure to ETS by children's caretakers and nicotine concentrations in children's hair $(199,317)$. However, other studies report only modest correlations between parental estimates of ETS exposure and nicotine and cotinine concentrations in children's hair (159, 170, 342). African-American children have higher cotinine concentrations in hair, cotinine concentrations in urine, and hair/urine concentration ratios than Caucasian children per self-reported cigarette smoked by the parents (170). Circumstantial evidence suggests that this may be related to hair pigmentation rather than ethnic differences in the pharmacokinetics of nicotine metabolism (72) since lower nicotine concentrations are found in white scalp hair compared to black scalp hair of the same subjects (331). Several studies report that white or fair hair has lower nicotine concentrations than black hair for similar levels of nicotine exposure $(331,335,338)$.

Strong correlations are found between nicotine concentrations in maternal and neonatal hair $(19.2 \pm 4.9$ vs. $2.4 \pm 0.9$ ng nicotine/mg hair; $r=0.49 ; p<0.001)$, and between cotinine concentrations in maternal and neonatal hair (6.3 $\pm 4.0 \mathrm{ng}$ vs. $2.8 \pm 0.8$ cotinine $/ \mathrm{mg}$ hair; $r=0.85 ; p=$ 0.0001) (158). However, a correlation between nicotine concentrations in maternal and newborn hair is not always 
evident (339). Nicotine concentrations in hair from newborns cannot be used as an indicator of fetal exposure to ETS. In neonates, only cotinine concentrations in hair are associated $(p<0.0001)$ with maternal cigarette consumption (315). Since fetal hair grows during the last three to four months of pregnancy, determination of nicotine and cotinine concentrations in neonatal hair is associated only with smoking during the third trimester.

\section{Breast milk}

Breast milk samples are usually obtained by hand expression $(205,343)$, or nipple aspiration using a breast pump $(187,205,306,312,344)$. Once obtained, breast milk can be a difficult matrix to analyse due to its variable lipid content, and may require complex extraction procedures prior to analysis $(178,187,253,343)$. Early studies report only the determination of nicotine concentrations in breast milk by GC-FID (178), GC-NPD (253), and GC-MS (187). More recent studies have determined both nicotine and cotinine concentrations by GC-NPD $(306,312,320,343$, 344) or HPLC-UV (205). Interest in the analysis of human breast milk has focused mainly on the transfer of nicotine and cotinine to the nursing infant via maternal breast milk from either smokers $(304,306,320,325,345)$ or ETSexposed non-smokers $(324,325,343)$, and transfer of nicotine during NRT to maternal milk (205).

Breast milk of smokers shows a wide concentration range of both nicotine $(0.5-140 \mathrm{ng}$ nicotine/mL) $(187,306,312$, $343,344)$ and cotinine $(<738 \mathrm{ng}$ cotinine $/ \mathrm{mL})(304,306$, $312,324,325,343,344)$. Strong linear correlations are found between the nicotine and cotinine concentrations in breast milk and the nicotine concentration in blood serum (306, 312), and between the cotinine concentration in breast milk and the cotinine concentration in serum (312). Breast milk:serum ratios are approximately 2.9 for nicotine and 0.8 for cotinine $(306,312)$. Nicotine concentrations in breast milk show diurnal profiles (344) similar to those observed in nicotine concentrations in blood (45). In contrast, cotinine concentrations in breast milk show little diurnal variation and are dependent on the extent of maternal smoking. The mean terminal half-life of nicotine in breast milk is $t_{1 / 2, \beta}=97 \pm 20 \mathrm{~min}$, similar to that of nicotine in maternal serum $\left(t_{1 / 2, \beta}=81 \pm 9 \mathrm{~min}\right)$ (312). Hence, exposure of the breast fed infants to nicotine and cotinine via maternal breast milk depends on daily cigarette consumption, individual smoking behaviour and, in the case of nicotine, time of smoking prior to nursing $(312,344)$.

Breast milk of non-smokers is reported to contain 1-7 ng nicotine/mL (343) and 2-63 ng cotinine/mL $(325,343)$. Significant correlations are found between the number of maternal cigarettes smoked and the nicotine concentration in breast milk $(320,344)$ and the cotinine concentration in breast milk $(304,320,324,344)$.

\section{Cervical mucus}

Samples of cervix mucus are usually collected by cervical flushing using sterile saline (345-347), although direct aspiration from the endocervix has been reported (153, 156). Cervical flushing appears to be a more efficient method of sample collection than using swab extracts
(345). The use of endocervical aspirates is difficult in epidemiological studies as many women cannot be expected to be in the preovulatory phase at the time of examination or are using oral contraceptive pills.

Nicotine concentrations in cervix mucus have been determined by both GC-MS (346) and RIA (153, 347); and cotinine concentrations by GC-NPD (348) and RIA (153, $156,345,347)$. In smokers, cervix mucus shows wide concentration ranges of 10-6,520 ng nicotine/mL cervical fluid $(153,156,345-347)$ and 8-6,662 ng cotinine/mL cervical fluid (153, 156, 345, 347, 348). However, both nicotine and cotinine concentrations are dependent on the method of sample collection; direct aspiration of smokers yields higher concentration ranges $(10-6,520 \mathrm{ng}$ nicotine/mL cervix mucus; $8-6,662 \mathrm{ng}$ cotinine/mL cervix mucus) compared to cervical flushing with saline (4-512 ng nicotine/mL cervix fluid; $2-167 \mathrm{ng}$ cotinine/mL cervix fluid) due to the dilution effect of the saline flush. Cotinine concentrations in cervix mucus are higher during the proliferative phase than the secretory luteal phase of the menstrual cycle and appear to be suppressed by use of oral contraception (348). Cotinine concentrations in cervix mucus and cotinine concentrations in urine are poorly correlated in all menstrual phases (348).

Nicotine and cotinine concentrations in cervix mucus are highly correlated ( $r=0.60 ; p<0.04)$, and both are correlated with the number of cigarettes smoked during the last $24 \mathrm{~h}$ prior to sample collection $(r=0.58$ and $r=0.57$, respectively) (345). Other studies confirm a strong association between smoking activity during the previous $24 \mathrm{~h}$ and cervix mucus concentrations of nicotine (153) and cotinine (348). Both cotinine and, especially, nicotine concentrations in cervix mucus of smokers are higher than in blood serum $(153,156,348)$. In non-smoking women, a highly significant association has been reported between self-reported exposure to ETS within the previous $24 \mathrm{~h}$ and nicotine concentrations in cervical lavages (346).

\section{Follicular fluid}

Cotinine concentrations have been reported in ovarian follicular fluids collected at the time of oocyte recovery during treatment for in vitro fertilization by RIA $(168,169)$ and FIA (167). Using RIA, follicular fluid contains $0.25-3,232 \mathrm{ng}$ cotinine/mL follicular fluid; concentrations are lowest in non-smokers $(4.2 \pm 2.0$, range $0.27-99.1 \mathrm{ng}$ cotinine $/ \mathrm{mL})$ and increase in women who either report exposure to ETS $(76.3 \pm 56.3 \mathrm{ng}$ cotinine/mL) or are current smokers $(710.4$ $\pm 128.2 \mathrm{ng}$ cotinine/mL follicular fluid) (168). Lower concentrations of $606 \pm 147 \mathrm{ng}$ cotinine/mL follicular fluid have been found using double antibody RIA (169).

\section{Fetal fluids}

Maternal cotinine concentrations above $25 \mathrm{ng}$ cotinine $/ \mathrm{mL}$ serum or above $250 \mathrm{ng}$ cotinine $/ \mathrm{mL}$ urine are invariably associated with detectable cotinine concentrations in fetal compartments (162). Cotinine accumulates in the fetal compartment as early as week 7 in both smokers and non-smokers exposed to ETS. First-trimester fetal fluids retrieved by transvaginal puncture contain $99 \pm 10 \mathrm{ng}$ cotinine $/ \mathrm{mL}$ coelomic fluid and $108 \pm 30 \mathrm{ng}$ cotinine/mL amniotic fluid. In 
smokers, positive linear correlations exist between cotinine concentrations in maternal urine and amniotic fluid. In second-trimester samples obtained by transabdominal puncture, higher cotinine concentrations are observed in smokers compared to non-smokers in both amniotic fluid (128 \pm 17 vs. $56 \pm 13 \mathrm{ng}$ cotinine $/ \mathrm{mL})$ and fetal serum ( $224 \pm 32$ vs. 66 $\pm 15 \mathrm{ng}$ cotinine $/ \mathrm{mL}$ ). Another study reports no dose-response relationships between smoking (determined in packs per day) and both nicotine and cotinine concentrtions in amniotic fluid (149). Both studies determined nicotine and cotinine concentrations by RIA.

Meconium (desquamated cells, mucus and bile that collect in the fetal bowel and is discharged shortly after birth) contains both cotinine and trans-3'-hydroxycotinine $(160,203)$. Although cotinine and trans-3'-hydroxycotinine concentrations in first day meconium samples appear to be higher in infants from women who smoke compared to infants from non-smokers (160), only very sparse data are available for the presence of nicotine metabolites in this matrix.

\section{Seminal plasma}

Seminal plasma is best obtained by masturbation after a period of sexual abstinence to increase the concentration of spermatozoa in the ejaculate. Following liquefaction and centrifugation to remove sperm pellets, nicotine concentrations in seminal plasma have been determined by RIA (155), while cotinine concentrations have been determined by RIA $(155,165,171,172,349,350)$, HPLC-UV at 262 nm (201), and HPLC-MS (209). Both nicotine and trans3 '-hydroxycotinine concentrations in seminal plasma have been determined by HPLC-MS (209). Nicotine metabolite concentrations in seminal plasma have been used to differentiate between smokers and non-smokers $(165,350)$, and as an indicator of ETS exposure (155). However, most studies have determined nicotine metabolite concentrations in seminal plasma as a marker of tobacco use during investigations of smoking on semen quality $(172,209,349)$ and sperm DNA damage $(171,201)$.

Nicotine and its principal metabolites, cotinine and trans3'-hydroxycotinine, have been found at concentrations of $93.1 \pm 31.0$ (range $0-124$ ) ng/mL, $279.8 \pm 155.0$ (range 78-655) $\mathrm{ng} / \mathrm{mL}$, and $37.5 \pm 7.0$ (range $8-113$ ) $\mathrm{ng} / \mathrm{mL}$ in seminal plasma, respectively (209). In smokers, strong correlations are found between seminal plasma and blood serum concentrations of cotinine $(r=0.974, p<0.005)$ and trans-3'-hydroxycotinine $(r=0.670 ; p<0.005)$, but not nicotine ( $r=0.333$; $p$ not significant) (209). Another study reports concentrations of 376-1,000 ng cotinine/mL seminal plasma (350). The cotinine concentration ratio between seminal plasma and serum is 1.6-2.1 $(155,209)$. Cotinine concentrations in seminal plasma are significantly $(p<$ $0.0001)$ higher in smokers $(252.9 \pm 37.0 \mathrm{ng}$ cotinine $/ \mathrm{mL})$ compared to non-smokers $(4.2 \pm 2.1 \mathrm{ng}$ cotinine $/ \mathrm{mL})$, and a significant correlation exists between the number of selfreported cigarettes smoked per day and cotinine concentrations in seminal plasma $(r=0.775 ; p<0.0001)(171)$. Similar results have been published in earlier studies (165, 209, 349). Cotinine concentrations in seminal plasma and blood plasma are also correlated $\left(r_{\mathrm{s}}=0.77\right)(172)$.

In non-smokers with self-reported exposure to ETS, seminal plasma concentrations of $12.4 \pm 10.9 \mathrm{ng}$ nicotine $/ \mathrm{mL}$ and $5.2 \pm 5.9 \mathrm{ng}$ cotinine/mL have been reported (155). No correlation was found between either nicotine or cotinine concentrations in seminal plasma and self-reported exposure to ETS. Other studies report significantly $(p<0.001)$ higher cotinine concentrations in seminal plasma of nonsmokers reporting exposure to ETS $(136 \pm 13$; range 100$170 \mathrm{ng}$ cotinine/mL) compared to non-smokers without exposure to ETS $(10 \pm 4.8$; range $0-50 \mathrm{ng}$ cotinine $/ \mathrm{mL})$ (350). The difference in cotinine concentrations found in both studies requires further confirmation.

\section{Sweat}

Nicotine secretion occurs in both apocrine and eccrine sweat (351). Determination of nicotine in sweat collected using commercially available sweat patches has been suggested as a non-invasive technique for monitoring of tobacco exposure (352). However, data currently available suggest that this method does not allow quantitative assessment of nicotine exposure in either smokers or non-smokers.

\section{Toenail}

One study reports the determination of toenail nicotine as a biomarker of tobacco smoke exposure (353). Toenail clippings (10-30 mg) were digested in $1 \mathrm{M} \mathrm{NaOH}$ at $50{ }^{\circ} \mathrm{C}$ prior to analysis by HPLC-ECD. In smoking women, nicotine concentrations in toenail (mean $2.01 \mathrm{ng}$ nicotine $/ \mathrm{mg}$ nail) show a significant correlation $(r=0.82 ; p<0.0001)$ with self-reported cigarette consumption. Nicotine concentrations in toenail of women reporting exposure to ETS (mean $0.28 \mathrm{ng}$ nicotine/mg nail) are significantly ( $p=$ 0.0006 ) higher than in women unexposed to ETS (mean $0.08 \mathrm{ng}$ nicotine/mg nail).

\section{Deciduous teeth}

Nicotine and cotinine are present in deciduous teeth and teeth may be a promising non-invasive matrix for assessment of cumulative ETS exposure during childhood (354). Based on sparse data derived from teeth from 35 children, nicotine concentrations in teeth are significantly higher in children from smoking parents compared to children with non-smoking parents. Cotinine concentrations, although present in children's deciduous teeth, do not show a statistically significant relationship to parental smoking.

\section{CONCLUDING REMARKS}

Recent advances in analytical methods have increased the sensitivity of detection of nicotine metabolites in various biological fluids and matrices and have revealed high interindividual variability in nicotine metabolism. It is tempting to speculate that the observed difference between AfricanAmericans and Caucasians in cotinine concentrations in blood $(72,170,246,247,249,261-264,267)$ and saliva $(3,238,243)$, and the distribution of nicotine metabolites in urine (72) may be influenced by both differences in smoking behaviour as well as genetic factors. Experimental studies show reduced nicotine $\mathrm{C}$-oxidation in AfricanAmericans due to the presence of an ethnic-specific 
$C Y P 2 A 6 * 17$ allele with reduced catalytic activity in vitro (355). Reduced cotinine/nicotine plasma ratios are also observed in African-American subjects either heterozygous or homozygous for the $C Y P 2 A 6^{*} 17$ allele after chewing a piece of nicotine-containing gum. If this is confirmed in future studies with smokers, determination of nicotine plus multiple phase I and phase II metabolites in 24-hour urine samples and calculation of results expressed as "nicotine equivalents", rather than single metabolites in blood, saliva and urine may provide the most accurate method to determine exposure to nicotine. However, determination of multiple nicotine metabolites in urine is complex and few laboratories are able to perform the required analysis. While this method has practical limitations, particularly compliance to collection of complete 24-hour urine samples, it may be the only method available to negate the effect of genetic factors and drug metabolism polymorphisms on the major pathways of nicotine metabolism. Outside of the clinical environment in which collection of 24-hour urine samples may be possible with good subject compliance, determination of the single nicotine metabolite, cotinine, in various biological fluids is likely to remain the most common approach to assess exposure to tobacco smoke in both smokers and nonsmokers.

\section{REFERENCES}

1. Jarvis, M.J., H. Tunstall-Pedoe, C. Feyerabend, C. Vesey, and Y. Saloojee: Comparison of tests used to distinguish smokers from nonsmokers; Am. J. Public Health 77 (1987) 1435-1438.

2. Patrick, D.L., A. Cheadle, D.C. Thompson, P. Diehr, T. Koepsell, and S. Kinne: The validity of self-reported smoking: a review and meta-analysis; Am. J. Resp. Crit. Care Med. 84 (1994) 1086-1093.

3. Boyd, N.R., R.A. Windsor, L.L. Perkins, and J.B. Lowe: Quality of measurement of smoking status by self-report and saliva cotinine among pregnant women; Matern. Child Health J. 2 (1998) 77-83.

4. Lindqvist, R., L. Lendahls, Ö. Tollbom, H. Åberg, and A. Håkansson: Smoking during pregnancy: comparison of self-reports and cotinine levels in 496 women; Acta Obstet. Gynecol. Scand. 81 (2002) 240-244.

5. Lee, P.N. and B.A. Forey: Misclassification of smoking habits as determined by cotinine or by repeated selfreport - a summary of evidence from 42 studies; J. Smoking-Related Dis. 6 (1995) 109-129.

6. Scherer, G. and E. Richter: Biomonitoring exposure to environmental tobacco smoke (ETS): a critical reappraisal; Human Exp. Toxicol. 16 (1997) 449-459.

7. Haley, N.J., C.M. Axelrod, and K.A. Tilton: Validation of self-reported smoking behavior: biochemical analysis of cotinine and thiocyanate; Am. J. Public Health 73 (1983) 1204-1207.

8. Van Vunakis, H., D.P. Tashkin, B. Rigas, M. Simmons, H.B Gjika, and V.A. Clark: Relative sensitivity and specificity of salivary and serum cotinine in identifying tobacco-smoking status of self-reported nonsmokers and smokers of tobacco and/or marijuana; Arch. Environ. Health 44 (1989) 53-58.

9. Tunstall-Pedoe, H., M. Woodward, and C.A. Brown: Tea drinking, passive smoking, smoking deception and serum cotinine in the Scottish Heart Health Study; J.
Clin. Epidemiol. 44 (1991) 1411-1414.

10. Murray, R.P., J.E. Connett, J.A. Istvan, M.A. Nides, and S. Rempel-Rossum: Relations of cotinine and carbon monoxide to self-reported smoking in a cohort of smokers and ex-smokers followed over 5 years; Nicotine Tob. Res. 4 (2002) 287-294.

11. Scherer, G.: Smoking behaviour and compensation: a review of the literature; Psychopharmacology (Berl.) 145 (1999) 1-20.

12. Haley, N.J., S.G. Colosimo, C.M. Axelrad, R. Harris, and D.W. Sepkovic: Biochemical validation of selfreported exposure to environmental tobacco smoke; Environ. Res. 49 (1989) 127-135.

13. Benowitz, N.L.: Cotinine as a biomarker of environmental tobacco smoke exposure; Epidemiol. Rev. 18 (1996) 188-204.

14. DeLorenze, G.N., M. Kharrazi, F.L. Kaufman, B. Eskenazim, and J.F. Bernert: Exposure to environmental tobacco smoke in pregnant women: The association between self-report and serum cotinine; Environ. Res. 90 (2002) 21-32.

15. Jarvis, M.J.: Trends in sales weighted tar, nicotine and carbon monoxide yields of UK cigarettes; Thorax 56 (2001) 960-963.

16. Wortley, P.M., R.S. Carabello, L.L. Pederson, and T.F. Pechacek: Exposure to secondhand smoke in the workplace: Serum cotinine by occupation; J. Occup. Environ. Med. 44 (2002) 503-509.

17. Seccareccia, F., P. Zuccaro, R. Pacifici, P. Meli, F. Pannozzo, K.M. Freeman, A. Santaquilani, S. Giampaoli: Serum cotinine as a marker of environmental tobacco smoke exposure in epidemiological studies: The experience of the MATISS project; Eur. J. Epidemiol. 18 (2003) 487-492.

18. Palmer, K.J., M.M. Buckley, and D. Faulds: Transdermal nicotine: A review of its pharmacodynamic and pharmacokinetic properties and therapeutic efficacy as an aid to smoking cessation. Drugs 44 (1992) 498-529.

19. Sutherland, G., M.A.H. Russell, J. Stapleton, C. Feyerabend, and O. Ferno: Nasal nicotine spray: A rapid nicotine delivery system; Psychopharmacology (Berl.) 108 (1992) 512-518

20. Guthrie, S.K., J.-K. Zubieta, L. Ohl, L. Ni, R.A. Koeppe, S. Minoshima, and E.F. Domino: Arterial/venous plasma nicotine concentrations following nicotine nasal spray; Eur. J. Clin. Pharmacol. 55 (1999) 639-643.

21. Ji, A.J. Jr., G.M. Lawson, R. Anderson, L.C. Dale, I.T. Croghan, and R.D. Hurt: A new gas chromatographymass spectrometry method for simultaneous determination of total and free trans-3'-hydroxycotinine and cotinine in the urine of subjects receiving transdermal nicotine; Clin. Chem. 45 (1999) 85-91.

22. Hurt, R.D., G.A. Croghan, S.D. Beede, T.D. Wolter, I.T. Crogan, and C.A. Patten: Nicotine patch therapy in 101 adolescent smokers; Arch. Pediatr. Adolesc. Med. 154 (2000) 31-37.

23. Schneider, N.G., R.E. Olmstead, M.A. Franzon, and E. Lunell: The nicotine inhaler. Clinical pharmacokinetics and comparison with other nicotine treatments; Clin. Pharmacokinet. 40 (2001) 661-684.

24. Miwa, K., Y. Miyagi, H. Asanoi, M. Fujita, and S. Sasayama: Augmentation of smoking cessation education by urinary cotinine measurements; Jpn. Circ. J. 57 (1993) 775-780. 
25. Trudeau, D.L., C. Isenhard, and D. Silversmith: Efficacy of smoking cessation strategies in a treatment program; J. Addict. Dis. 14 (1995) 109-116.

26. Secker-Walker, R.H., P.M. Vacek, B.S. Flynn, and P.B. Mead: Exhaled carbon monoxide and urinary cotinine as measures of smoking in pregnancy; Addict. Behav. 22 (1997) 671-684.

27. Davis, R.A., M.F. Stiles, J.D. deBethizy, and J.H. Reynolds: Dietary nicotine: a source of urinary cotinine; Food Chem. Toxicol. 29 (1991) 821-827.

28. Siegmund, B., E. Leitner, and W. Pfannhauser: Determination of nicotine content of various edible nightshades (Solanaceae) and their products and estimation of the associated dietary nicotine intake; J. Agric. Food Chem. 47 (1999) 3113-3120.

29. Armstrong, D.W., X. Wang and N. Ercal: Enantiometric composition of nicotine in smokeless tobacco, medicinal products and commercial reagents; Chirality 10 (1998) 587-591.

30. Pool, W.F., C.S. Godin, and P.A. Crooks: Nicotine racemization during tobacco smoking; The Toxicologist 5 (1985) 232.

31. Armitage, A.K. and D.M. Turner: Absorption of nicotine in cigarette and cigar smoke through the oral mucosa; Nature 226 (1970) 1231-1232.

32. Beckett, A.H., J.W. Gorrod, and P. Jenner: A possible relationship between $\mathrm{pKa}_{1}$ and lipid solubility and the amounts excreted in urine of some tobacco alkaloids given to man; J. Pharm. Pharmac. 24 (1972) 115-120.

33. Armitage, A., C. Dollery, T. Houseman, E. Kohner, P.J. Lewis, and D. Turner: Absorption of nicotine from small cigars; Clin. Pharmacol. Ther. 23 (1978) 143-151.

34. Armitage, A.K., M. Dixon, B.E. Frost, D.C. Mariner, and N.M. Sinclair: The effect of tobacco blend additives on the retention of nicotine and solanesol in the human respiratory tract and on subsequent plasma nicotine concentrations during cigarette smoking; Chem. Res. Toxicol. 17 (2004) 537-544.

35. Wald, N.J., M. Idle, J. Boreham, A. Bailey, and H. Van Vunakis: Serum cotinine levels in pipe smokers: Evidence against nicotine as a cause of coronary heart disease; Lancet 2 (1981) 775-777.

36. Gori, G.B., N.L. Benowitz, and C.J. Lynch: Mouth verses deep airways absorption of nicotine in cigarette smokers. Pharmacol; Biochem. Behav. 25 (1986) 1181-1184.

37. Rieben, F.W.: Smoking behaviour and increase in nicotine and carboxyhaemoglobin in venous blood; Clin. Invest. 70 (1992) 335-342.

38. Benowitz, N.L.: Pharmacologic aspects of cigarette smoking and nicotine addiction; N. Engl. J. Med. 319 (1988) 1318-1330.

39. Armitage, A.K., C.T. Dollery, C.F. George, T.H. Houseman, P.J. Lewis, and D.M. Turner: Absorption and metabolism of nicotine from cigarettes; Br. Med. J. 4 (1975) 313-316.

40. Henningfield, J.E., E.D. London, and N.L. Benowitz: Arterial-venous differences in plasma concentrations of nicotine after cigarette smoking; JAMA 263 (1990) 2049-2050.

41. Lunell, E., L. Molander, K. Ekberg, and J. Wahren: Site of nicotine absorption from a vapour inhaler comparison with cigarette smoking; Eur. J. Pharmacol. 55 (2000) 737-741.
42. Herning, R.I., R.T. Jones, N.L. Benowitz, and A.H. Mines: How a cigarette is smoked determines nicotine blood levels; Clin. Pharmacol. Ther. 33 (1983) 84-90.

43. Adlkofer, F., G. Scherer, A. Biber, W.-D. Heller, P.N. Lee, and H. Schievelbein: Consistency of nicotine intake in smokers of cigarettes with varying nicotine yields; in: Nicotine, Smoking and the Low Tar Programme, edited by N. Wald and P. Froggart; Oxford University Press, Oxford, 1989, pp. 116-130.

44. Byrd, G.D., J.H. Robinson, W.S. Caldwell, and J.D. deBethizy: Comparison of measured and FTC-predicted nicotine uptake in smokers; Psychopharmacology 122 (1995) 95-103.

45. Benowitz, N.L., F. Kuyt, and P. Jacob III.: Circadian blood nicotine concentrations during cigarette smoking; Clin. Pharmacol. Ther. 32 (1982) 758-764.

46. Russell, M.A.H., C. Wilson, U.A. Patel, C. Feyerabend, and P.V. Cole: Plasma nicotine levels after smoking cigarettes with high, medium and low nicotine yields; Br. Med. J. 2 (1975) 414-416.

47. Russell, M.A.H., M. Jarvis, R. Iyer, and C. Feyerabend: Relationship of nicotine yield of cigarettes to blood nicotine concentration in smokers; Br. Med. J. (1980) 972-976.

48. Benowitz, N.L., S.M. Hall, R.L. Herning, P. Jacob, III., R.T. Jones, and A.-L. Osman: Smokers of low-yield cigarettes do not consume less nicotine; N. Engl. J. Med. 309 (1983) 139-142.

49. Ebert, R.V., K.E. McNabb, K.T. McCuskar, and S.L. Snow: Amount of nicotine and carbon monoxide inhaled by smokers of low-tar, low-nicotine cigarettes; JAMA 250 (1983) 2840-2842.

50. Gori, G.B. and C.J. Lynch: Smoker intake from cigarettes in the 1-mg Federal Trade Commission tar class; Regul. Toxicol. Pharmacol. 3 (1983) 110-120.

51. Gori, G.B. and C.J. Lynch: Analytical cigarette yields as predictors of smoke bioavailability; Regul. Toxicol. Pharmacol. 5 (1985) 314-326.

52. Benowitz, N.L. and P. Jacob III.: Daily intake of nicotine during cigarette smoking; Clin. Pharmacol. Ther. 35 (1984) 499-504.

53. Feyerabend, C., R.M.J. Ings, and M.A.H. Russell: Nicotine pharmacokinetics and its application to intake from smoking; Br. J. clin. Pharmac. 19 (1985) 239-247.

54. Bridges, R.B., J.W. Humble, J.A. Turbek, and S.R. Rehm: Smoking history, cigarette yield and smoking behavior as determinants of smoke exposure; Eur. J. Respi. Dis. 69 (Suppl. 146) (1986) 129-137.

55. Bridges, R.B., J.G. Combs, J.W. Humble, J.A. Turbek, S.R. Rehm, and N.J. Haley: Population characteristics and cigarette yield as determinants of smoke exposure; Pharmacol. Biochem. Behav. 37 (1990) 17-28.

56. Zacny, J.P. and M.L. Stitzer: Cigarette brand-switching: effects on smoke exposure and smoking behavior; J. Pharmacol. Expt. Ther. 246 (1988) 619-627.

57. Kolonen, S.A. and E.V.J. Puhakainen: Assessment of the automated colorimetric and the high-performance liquid chromatographic methods for nicotine intake by urine samples of smokers' smoking low- and mediumyield cigarettes; Clin. Chim. Acta 196 (1991) 159-166.

58. Bruckert, E., N. Jacob, L. Lamaire, J. Truffert, F. Percheron, and J.L. de Gennes: Relationship between smoking status and serum lipids in a hyperlipidemic population and analysis of possible confounding factors; Clin. Chem. 38 (1992) 1698-1705. 
59. Woodward, M. and H. Tunstall-Pedoe: Self-titration of nicotine: evidence from the Scottish Heart Health Study; Addiction 88 (1993) 821-830.

60. Coultas, D.B., C.A. Stidley, and J.M. Samet: Cigarette yields of tar and nicotine and markers of exposure to tobacco smoke; Am. Rev. Respir. Dis. 148 (1993) 435-440.

61. Hee, J., F. Callais, I. Momas, A.M. Laurent, S. Min, P. Molinier, M. Chastagnier, J.R. Claude, and B. Festy: Smokers' behavior and exposure according to cigarette yield and smoking experience; Pharmacol. Biochem. Behav. 52 (1995) 195-203.

62. Andersson, G., E.K. Vala, and M. Curvall: The influence of cigarette consumption and smoking machine yields of tar and nicotine on the nicotine uptake and oral mucosal lesions in smokers; J. Oral Pathol. Med. 26 (1997) 117-123.

63. Byrd, G.D., R.A. Davies, W.S. Caldwell, J.H. Robinson, and J.D. deBethizy: A further study of FTC yield and nicotine absorption in smokers. Psychopharmacology 139 (1998) 291-229.

64. Jarvis, M.J., R. Boreham, P. Primatesta, C. Feyerabend, and A. Bryant: Nicotine yield from machine-smoked cigarettes and nicotine intakes in smokers: Evidence from a representative population survey; J. Natl. Cancer Inst. 93 (2001) 134-138.

65. Ueda, K., I. Kawachi, M. Nakmura, H. Nogami, N. Shirokawa, S. Masui, A. Okayama, and A. Oshima: Cigarette nicotine yields and nicotine intake among Japanese male smokers; Tobacco Control 11 (2002) $55-60$.

66. Nakazawa, A., M. Shigeta, and K. Ozasa: Smoking cigarettes of low nicotine yield does not reduce nicotine intake as expected: A study of nicotine dependency in Japanese males; BMC Public Health 4 (2004) 28.

67. Federal Trade Commission: Cigarettes. Testing for tar and nicotine content - statements of considerations; Fed. Regist. 32 (1967) 11178.

68. Sato, H. and S. Araki: Yields and daily consumption of cigarettes in Japan in 1969-1966; J. Epidemiol. 10 (2000) 7-15.

69. National Cancer Institute: Risks associated with cigarette smoking with low machine-measured yields of tar and nicotine. Smoking and Tobacco Control Monograph No. 13. Bethesda, MD: U.S. Department of Health and Human Services, National Institute of Health, National Cancer Institute; NIH Publ. No. 02-5074, 2001.

70. Benowitz, N.L., P. Jacob, III., C. Denaro, and R. Jenkins: Stable isotope studies of nicotine kinetics and bioavailability; Clin. Pharmacol. Ther. 49 (1991) 270-277.

71. Benowitz, N.L. and P. Jacob, III.: Metabolism of nicotine to cotinine studied by a dual stable isotope method; Clin. Pharmacol. Ther. 56 (1994) 483-493.

72. Pérez-Stable, E.J., B. Herrera, P. Jacob III., and N.L. Benowitz: Nicotine metabolism and intake in black and white smokers; JAMA 280 (1998) 152-156.

73. Benowitz, N.L., E.J. Pérez-Stable, B. Herrera, and P. Jacob III.: Slower metabolism and reduced intake of nicotine from cigarette smoking in Chinese-Americans; J. Natl. Cancer Inst. 94 (2002) 108-115.

74. Byrd, G.D., K.-M. Chang, J.M. Greene, and J.D. deBethizy: Evidence for urinary excretion of glucuronide conjugates of nicotine, cotinine and trans-3'hydroxycotinine in smokers; Drug Metab. Dispos. 20 (1992) 192-197.
75. Curvall, M., E. Kazemi-Vala, and G. Englund: Conjugation pathways in nicotine metabolism; in: Effects of Nicotine on Biological Systems, edited by F. Adlkofer and K. Thurau, Birkhäuser Verlag, Basel, 1991, pp. 69-75.

76. Seaton, M.J., E.S. Vesell, H. Luo, and E.M. Hawes: Identification of radiolabeled metabolites of nicotine in rat bile. Synthesis of S-(-)-nicotine $N$-glucuronide and direct separation of nicotine-derived conjugates using high-performance liquid chromatography; J. Chromatogr. 621 (1993) 49-53.

77. Caldwell, W.S., J.M. Greene, G.D. Byrd, K.M. Chang, M.S. Uhrig, J.D. deBethizy, P.A. Crooks, B.S. Bhatti, and R.M. Riggs: Characterization of the glucuronide conjugate of cotinine: A previously unidentified major metabolite of nicotine in smokers' urine; Chem. Res. Toxicol. 5 (1992) 280-285.

78. Schepers, G., D. Demetriou, K. Rustemeier, P. Voncken, and B. Diehl: Nicotine phase 2 metabolites in human urine - structure of metabolically formed trans3'-hydroxycotinine glucuronide; Med. Sci. Res. 20 (1992) 863-865.

79. Gorrod, J.W. and G. Schepers: Biotransformation of nicotine in mammalian systems; in: Analytical Determination of Nicotine and Related Compounds and their Metabolites, edited by J.W. Gorrod and P. Jacob, III., Elsevier, Amsterdam, 1999, pp. 45-67.

80. De Schepper, P.J., A. Van Heecken, P. Daenens, and J.M. Van Rossum: Kinetics of cotinine after oral and intravenous administration; Eur. J. Clin. Pharmacol. 31 (1987) 583-588.

81. Scherer, G., L. Jarczyk, W.-D. Heller, A. Biber, G.B. Neurath, and F. Adlkofer: Pharmacokinetics of nicotine, cotinine and 3'-hydroxycotinine in cigarette smokers. Klin. Wochenschr. 66 (Suppl. XI)(1988) 5-11.

82. Kyerematen, G.A., M.L. Morgan, B. Chattopadhyay, J.D. deBethizy, and E.S. Vesell: Disposition of nicotine and eight metabolites in smokers and nonsmokers: Identification in smokers of two metabolites that are longer lived than cotinine; Clin. Pharmacol. Ther. 48 (1990) 641-651.

83. Curvall, M., E. Kazemi-Vala, C.R. Enzell, and J. Wahren: Simulation and evaluation of nicotine intake during passive smoking: Cotinine measurements in body fluids of nonsmokers given intraveneous infusions of nicotine; Clin. Pharmacol. Ther. 47 (1990) 42-49.

84. Curvall, M., C.-E. Elwin, E. Kazemi-Vala, C. Warholm, and C.R. Enzell: The pharmacokinetics of cotinine in plasma and saliva from non-smoking healthy volunteers; Eur. J. Clin. Pharmacol. 38 (1990) 281-287.

85. Kyerematen, G.A. and E.S. Vesell: Metabolism of nicotine; Drug Metab. Rev. 23 (1991) 3-41.

86. Benowitz, N.L. and P. Jacob, III.: Effects of cigarette smoking and carbon monoxide on nicotine and cotinine metabolism; Clin. Pharmacol. Ther. 67 (2000) 653-659.

87. Benowitz, N.L. and P. Jacob, III.: Trans-3'-hydroxycotinine: disposition kinetics, effects and plasma levels during cigarette smoking; J. Clin. Pharmacol. 51 (2001) 53-59.

88. Benowitz, N.L., E.J. Perez-Stable, I. Fong, G. Modin, B. Herrera, and P. Jacob, III.: Ethnic differences in $N$ glucuronidation of nicotine and cotinine; J. Pharmacol. Exp. Ther. 291 (1999) 1196-1203.

89. Zevin, S., P. Jacob, III., P. Geppetti, and N.L. Benowitz: Clinical pharmacology of oral cotinine; Drug 
Alcohol Depend. 60 (2000) 13-18.

90. Benowitz, N.L. and P. Jacob, III.: Nicotine and cotinine elimination pharmacokinetics in smokers and nonsmokers; Clin. Pharmacol. Ther. 53 (1993) 316-323.

91. Langmann, P., A. Bienert, M. Zilly, T. Väth, E. Richter, and H. Klinker: Influence of smoking on cotinine and caffeine plasma levels in patients with alcoholic liver cirrhosis; Eur. J. Med. Res. 5 (2000) 217-221.

92. Molander, L., A. Hansson, E. Lunell, L. Alainentalo, M. Hoffmann, and R. Larsson: Pharmacokinetics of nicotine in kidney failure; Clin. Pharmacol. Ther. 68 (2000) 250-260.

93. Benowitz, N.L., P. Jacob, III., I. Fong, and S. Gupta: Nicotine metabolic profile in man: comparison of cigarette smoking and transdermal nicotine; J. Pharmacol. Exp. Ther. 268 (1994) 296-303.

94. Molander, L., A. Hansson, and E. Lunell: Pharmacokinetics of nicotine in healthy elderly people; Clin. Pharmacol. Ther. 69 (2001) 57-65.

95. Dempsey, D., P. Jacob, III., and N.L. Benowitz: Accelerated metabolism of nicotine and cotinine in pregnant smokers; J. Pharmacol. Expt. Ther. 301 (2002) 594-598.

96. Dempsey, D., P. Jacob, III., and N.L. Benowitz: Nicotine metabolism and elimination kinetics in newborns; Clin. Pharmacol. Ther. 67 (2000) 458-465.

97. Hecht, S.S., S.G. Carmella, and S.E. Murphy: Effects of watercress consumption on urinary metabolites of nicotine in smokers; Cancer Epidemiol. Biomarkers Prev. 8 (1999) 907-913.

98. Meger, M., I. Meger-Kossien, A. Schuler-Metz, D. Janket, and G. Scherer: Simultaneous determination of nicotine and eight metabolites in urine of smokers using liquid chromatography - tandem mass spectrometry; J. Chromatogr. B 778 (2002) 251-261.

99. Benowitz, N.L., B. Herrera, and P. Jacob, III.: Mentholated cigarette smoking inhibits nicotine metabolism; J. Pharmacol. Expt. Ther. 310 (2004) 1208-1215.

100. Flammang, A.M., H.V. Gelboin, T. Aoyama, F.J. Gonzales, and G.D. McCoy: Nicotine metabolism by cDNA-expressed human cytochrome P-450s; Biochem. Arch. 8 (1992) 1-8.

101. McCracken, N.W., S. Cholerton, and J.R. Idle: Cotinine formation by cDNA-expressed human cytochromes P450; Med. Sci. Res. 20 (1992) 877-878.

102. Nakajima, M., T. Yamamoto, K. Nunoya, T. Yokoi, K. Nagashima, K. Inoue, Y. Funae, N. Shimada, T. Kamataki, and Y. Kuroiwa: Role of human cytochrome P4502A6 in C-oxidation of nicotine; Drug Metab. Dispos. 24 (1996) 1212-1217.

103. Bao, Z., T. Su, X. Ding, and J.-Y. Hong: Metabolism of nicotine and cotinine by human cytochrome P450 2A13 (CYP2A13); $13^{\text {th }}$ International Symposium on Microsomes and Drug Oxidations, July 10-14 2000, Stressa, Italy, Abstract 137.

104. Messina, E.S., R.F. Tyndale, and E.M. Sellers: A major role for CYP2A6 in nicotine C-oxidation by human liver microsomes; J. Pharmacol. Exp. Ther. 282 (1997) 1608-1614.

105. Yamazaki, H., K. Inoue, M. Hashimoto, and T. Shimada: Roles of CYP2A6 and CYP2B6 in nicotine C-oxidation by human liver microsomes; Arch. Toxicol. 73 (1999) 65-70.

106. Nakajima, M., T. Yamamoto, K. Nunoya, T. Yokoi, K. Nagashima, K. Inoue, Y. Funae, N. Shimada, T.
Kamataki, and Y. Kuroiwa: Characterization of CYP2A6 involved in 3'-hydroxylation of cotinine in human liver microsomes; J. Pharmacol. Exp. Ther. 277 (1996) 1010-1015.

107. Benowitz, N.L., P. Jacob, III., and E. Perez-Stable: CYP2D6 phenotype and the metabolism of nicotine and cotinine; Pharmacogenetics 6 (1996) 239-242.

108. Marez, D., M. Legrand, N. Sabbagh, J.-M. LoGuidice, C. Spire, J.-J. Lafitte, U.A. Meyer, and F. Broly: Polymorphism of the cytochrome P450 CYP2D6 gene in a European population: characterization of 48 mutations and 53 alleles, their frequencies and evolution; Pharmacogenetics 7 (1997) 193-202.

109. Lang, T., K. Klein, J. Fisher, A.K. Nüssler, P. Neuhaus, U. Hofmann, M. Eichelbaum, M. Schwab, and U.M. Zanger: Extensive genetic polymorphism in the human CYP2B6 gene with impact on expression and function in human liver; Pharmacogenetics 11 (2001) 399-415.

110. Tricker, A.R.: Nicotine metabolism, human drug metabolism polymorphisms and smoking behaviour; Toxicology 183 (2003) 151-173.

111.Zhang, X., Y. Chen, Y. Liu, X. Ren, Q.Y. Zhang, M. Caggana, and $X$. Ding: Single nucleotide polymorphisms of the human cyp2a13 gene: Evidence for a null allele; Drug Metab. Dispos. 30 (2003) 1081-1085.

112. Cashman, J.R., S.B. Park, Z.C. Yang, S.A. Wrighton, P. Jacob, III., and N.L. Benowitz: Metabolism of nicotine by human liver microsomes: Stereoselective formation of trans-nicotine $N$ '-oxide; Chem. Res. Toxicol. 5 (1992) 639-646.

113. Kuehl, G. and S.E. Murphy: N-Glucuronidation of nicotine and cotinine by human liver microsomes and heterologously expressed UDP-glucuronosyltransferases; Drug Metab. Dispos. 31 (2003) 1361-1368.

114. Benowitz, N.L. and P. Jacob, III.: Individual differences in nicotine kinetics and metabolism in humans; NIDA Res. Monogr. 173 (1997) 48-64.

115. Sellers, E.M.: Pharmacogenetics and ethnoracial differences in smoking; JAMA 280 (1998) 179-180.

116. Russell, M.A.H. and C. Feyerabend: Cigarette smoking: a dependence on high-nicotine boli; Drug Metab. Rev. 8 (1978) 29-57.

117. Benowitz, N.L., O.F. Pomerleau, C.S. Pomerleau, and P. Jacob, III.: Nicotine metabolite ratio as a predictor of cigarette consumption; Nicotine Tob. Res. 5 (2003) 621-624.

118. Davis, R.A. and M. Curvall: Determination of nicotine and its metabolites in biological fluids: in vivo studies; in: Analytical Determination of Nicotine and Related Compounds and their Metabolites, edited by J.W. Gorrod and P. Jacob, III., Elsevier, Amsterdam, 1999, pp. 583-644.

119. Smith, R.F., H.M. Mather, and G.A. Ellard: Assessment of simple colorimetric procedures to determine smoking status of diabetic subjects; Clin. Chem. 44 (1998) 275-280.

120. Langone, J.J., H.B. Gjika, and H. Van Vunakis: Use of immunoassay techniques for the determination of nicotine and its metabolites; in: Analytical Determination of Nicotine and Related Compounds and their Metabolites, edited by J.W. Gorrod and P. Jacob, III., Elsevier, Amsterdam, 1999, pp. 265-284.

121. Jacob, P., III and G.D. Byrd: Use of gas chromatographic and mass spectrometric techniques for the determination of nicotine and its metabolites; in: Analytical Determination of Nicotine and Related Compounds and 
their Metabolites, edited by J.W. Gorrod and P. Jacob, III., Elsevier, Amsterdam, 1999, pp. 191-224.

122. Crooks, P.A. and G.D. Byrd: Use of high performance liquid chromatographic-mass spectrometric (LC-MS) techniques for the determination of nicotine and its metabolites; in: Analytical Determination of Nicotine and Related Compounds and their Metabolites, edited by J.W. Gorrod and P. Jacob, III., Elsevier, Amsterdam, 1999, pp. 225-264.

123. Bieber, A., G. Scherer, I. Hoepfner, F. Adlkofer, W.-D. Heller, J.E. Haddow, and G.J. Knight: The determination of nicotine and cotinine in human serum and urine: an interlaboratory study; Toxicol. Lett. 35 (1987) $45-52$.

124. Feyerabend, C. and M.A.H. Russell: Assay of nicotine in biological materials: sources of contamination and their elimination; J. Pharm. Pharmacol. 32 (1980) 178-181.

125. Curvall, M., E. Kazemi-Vala, and C.R. Enzell: Simultaneous determination of nicotine and cotinine in plasma using capillary column gas chromatography with nitrogen-sensitive detection; J. Chromatogr. B 232 (1982) 283-293.

126. Jones, D., M. Curvall, L. Abrahamsson, E. KazemiVala, and C. Enzell: Quantitative analysis of plasma nicotine using selected ion monitoring at high resolution. Biomed. Mass Spectrometry 9 (1982) 539-545.

127. Teeuwen, H.W.A., R.J.W. Aalders, and J.M. Van Rossum: Simultaneous estimation of nicotine and cotinine levels in biological fluids using high-resolution capillary-column gas chromatography combined with solid phase extraction work-up; Molecular Biol. Reports 13 (1989) 165-175.

128. Jacob, P. III., L. Yu, M. Wilson, and N.L. Benowitz: Selected ion monitoring method for determination of nicotine, cotinine and deuterium-labeled analogs: absence of an isotope effect in the clearance of $(S)$ nicotine- $3^{\prime}, 3^{\prime}-\mathrm{d}_{2}$ in humans; Biol. Mass Spectrom. 20 (1991) 247-252.

129. Peach, H., G.A. Ellard, P.J. Jenner, and R.W. Morris: A simple, inexpensive urine test of smoking; Thorax 40 (1985) 351-357.

130. Ubbink, J.B., J. Lagendijk, and W.H.H. Vermaak: Simple high-performance liquid chromatographic method to verify the direct barbituric acid assay for urinary cotinine; J. Chromatogr. 620 (1993) 254-259.

131. Barlow, R.D., R.B. Stone, N.J. Wald, and E.V.J. Puhakainen: The direct barbituric acid assay for nicotine metabolites in urine: a simple colorimetric test for the routine assessment of smoking status and cigarette smoke intake; Clin. Chim. Acta 165 (1987) 45-52.

132. Barlow, R.D., P.A. Thompson and R.B. Stone: Simultaneous determination of nicotine, cotinine and five additional nicotine metabolites in the urine of smokers using pre-column derivatization and high-performance liquid chromatography; J. Chromatogr. 419 (1987) 375-380.

133. Moore, J., M. Greenwood, and N. Sinclair: Automation of a high-performance liquid chromatographic assay for the determination of nicotine, cotinine and 3'hydroxycotinine in human urine; J. Pharm. Biomed. Anal. 8 (1990) 1051-1054.

134. Puhakainen, E.V.J, R.D. Barlow, and J.T. Salonen: An automated colorimetric assay for urine nicotine metabolites: a suitable alternative to cotinine assays for the asssessment of smoking status; Clin. Chim. Acta 170
(1987) 255-262.

135. Pickert, A., T. Lingenfelser, C. Pickert, N. Birbaumer, D. Overkamp, and M. Eggstein: Comparison of a mechanized version of the "König" reaction and a fluorescence polarization immunoassay for the determination of nicotine metabolites in urine; Clin. Chem. Acta 217 (1993) 143-152.

136. Cope, G., P. Nayyar, R. Holder, J. Gibbons, and R. Bunce: A simple near-patient test for nicotine and its metabolites in urine to assess smoking habit; Clin. Chim. Acta 256 (1996) 135-149.

137. Parviainen, M.T. and R.D. Barlow: Assessment of exposure to environmental tobacco smoke using a highperformance liquid chromatographic method for the simultaneous determination of nicotine and two of its metabolites in urine; J. Chromatogr. 431 (1988) 216-221.

138. Rustemeier, K., D. Demetriou, G. Schepers, and P. Voncken: High-performance liquid chromatographic determination of nicotine and its urinary metabolites via their 1,3-diethyl-2-thiobarbituric acid derivatives; J. Chromatogr. 613 (1993) 95-103.

139. Chambers, K.L., G.A. Ellard, A.T. Hewson, and R.F. Smith: Urine test for the assessment of smoking status; Br. J. Biomed. Sci. 58 (2001) 61-65.

140. Langone, J.J., H.B. Gjika, and H. Van Vunakis: Nicotine and its metabolites. Radioimmunoassays for nicotine and cotinine; Biochemistry 12 (1973) 5025-5030.

141. Langone, J.J. and H. Van Vunakis: Radioimmunoassay of nicotine, cotinine and $\gamma$-(-pyridyl)- $\gamma$-oxo- $N$ methylbutyramide; Methods Enzymol. 84 (1982) 628-640.

142. Knight, G.J., P. Wylie, M.S. Holman, and J.E. Haddow: Improved ${ }^{125} \mathrm{I}$ radioimmunoassay for cotinine by selective removal of bridge antibodies; Clin. Chem. 31 (1985) 118-121.

143. Bjercke, R.J., G. Cook, N. Rychlik, H.B. Gjika, H. Van Vunakis, and J.J. Langone: Stereospecific monoclonal antibodies to nicotine and cotinine and their use in enzyme-linked immunosorbent assays; J. Immunol. Methods 90 (1986) 203-213.

144. Langone, J.J., G. Cook, R.J. Bjercke, and M.H. Lifschitz: Monoclonal antibody ELISA for cotinine in saliva and urine of active and passive smokers; J. Immunol. Methods 114 (1988) 73-78.

145. Benkirane, S., A. Nicolas, M.-M. Galteau, and G. Siest: Highly sensitive immunoassays for the determination of cotinine in serum and saliva. Comparison between RIA and an avidin-biotin ELISA; Eur. J. Clin. Chem. Clin. Biochem. 29 (1991) 405-410.

146. Hansel, M.C., F.J. Rowell, J. Landon, and A.M. Sidki: Single-reagent polarisation fluoroimmunoassay for cotinine (a nicotine metabolite) in urine; Ann. Clin. Biochem. 5 (1986) 596-602.

147. Alterman, A.I., P. Gariti, and R.S. Niedbala: Varying results for immunoassay screening kits for cotinine level; Psychol. Addict. Behav. 16 (2002) 256-259.

148. Niedbala, R.S., N. Haley, S. Kardos, and K. Kardos: Automated homogenous immunoassay analysis of cotinine in urine; J. Anal. Toxicol. 26 (2002) 166-170.

149. Van Vunakis, H., J.J. Langone, and A. Milunsky: Nicotine and cotinine in the amniotic fluid of smokers in the second trimester of pregnancy; Am. J. Obstet. Gynecol. 120 (1974) 64-66.

150. Haines, C.F., Jr., D.K. Mahajan, D. Miljiković, M. 
Miljiković, and E.S. Vesell: Radioimmunoassay of plasma nicotine in habituated and naive smokers; Clin. Pharmacol. Ther. 16 (1974) 1083-1090.

151. Castro, A., N. Monji, H. Ali, M. Yi, E.R. Bowman, and H. McKennis, Jr.: Nicotine antibodies: comparison of ligand specificities of antibodies produced against two nicotine conjugates; Eur. J. Biochem. 104 (1980) 331-340.

152. Hill, P. and H. Marquardt: Plasma and urine changes after smoking different brands of cigarettes; Clin. Pharm. 27 (1980) 652-658.

153. Hellberg, D., S. Nilsson, N.J. Haley, D. Hoffman, and E. Wynder: Smoking and cervical intraepithelial neoplasia: nicotine and cotinine in serum and cervical mucus in smokers and nonsmokers; Am. J. Obstet. Gynecol. 158 (1988) 910-913.

154. Van Vunakis, H., H.B. Gijka, and J.J. Langone: Radioimunnoassay for nicotine and cotinine; in: Environmental carcinogens-methods of analysis and exposure measurement, Vol. 12: International Agency for Research on Cancer, edited by B. Seifert, H. van der Wiel, B. Bodet, and I.K. O’Neill, Lyon, 1993, pp. 293-299.

155. Pacifici, R., I. Altiere, L. Gandini, A. Lenzi, A.R. Passa, S. Pichini, M. Rosa, P. Zuccaro, and F. Dondero: Environmental tobacco smoke: nicotine and cotinine in semen; Environ. Res. 68 (1995) 69-72.

156. Sasson, I.M., N.J. Haley., D. Hoffmann, E.L. Wynder, D. Hellberg, and S. Nilsson: Cigarette smoking and neoplasia of the uterine cervix: smoker constituents in cervical mucus; N. Engl. J. Med. 312 (1985) 315-316.

157. Haley, N.J. and D. Hoffmann: Analysis for nicotine and cotinine in hair to determine cigarette smoker status; Clin. Chem. 31 (1985) 1598-1600.

158. Eliopoulos, C., J. Klein, K. Phan, B. Knie, M. Greenwald, D. Chitayat, and G. Koren: Hair concentrations of nicotine and cotinine in women and their newborn infants; JAMA 271 (1994) 621-623.

159. Woodruff, S.I., T.L. Conway, C.C. Edwards, and M.F. Hovell: Acceptability and validity of hair collection from Latino children to assess exposure to environmental tobacco smoke; Nicotine Tob. Res. 5 (2003) 375-385.

160. Ostrea, E.M., D.K. Knapp, A. Romero, M. Montes, and A.R. Ostrea: Meconium analysis to assess fetal exposure to nicotine by active and passive maternal smoking; J. Pediatr. 124 (1994) 471-476.

161. Heinrich-Ramm, R., R. Wegner, A.H. Garde, and X. Baur: Cotinine excretion (tobacco smoke biomarker) of smokers and nonsmokers: comprison of GC/MS and RIA results; Int. J. Hyg. Environ. Health 205 (2002) 493-499.

162. Jauniaux, E., B. Gulbis, G. Acharya, P. Thiry, and C. Rodeck: Maternal tobacco exposure and cotinine levels in fetal fluids in the first half of pregnancy; Obstet. Gynecol. 93 (1999) 25-29.

163. Bjercke, R.J., G. Cook, and J.J. Langone: Comparison of monoclonal and polyclonal antibodies to cotinine in nonisotopic and isotopic immunoassays; J. Immunol. Methods 96 (1987) 239-246.

164. Perkins, S.L., J.F. Livesey, E.A. Escares, J.M. Belcher, and D.K. Dudley: High-performance liquid-chromatographic method compared with a modified radioimmunoassay of cotinine in plasma; Clin. Chem. 37 (1991) 1989-1992.

165. Vine, M.F., B.S. Hulka, B.H. Margolin, Y.K. Truong,
P.-C. Hu, M.H. Schramm, J.D. Griffith, M. McCann, and R.B. Everson: Cotinine concentrations in semen, urine and blood of smokers and nonsmokers; Am. J. Public Health 83 (1993) 1335-1338.

166. Nafstad, P., J. Kongerud, G. Botton, P. Urdal, T. Silsand, B.S. Pedersen, and J.J.K. Jaakkola: Fetal exposure to tobacco smoke products, a comparison between self-reported maternal smoking and concentrations of cotinine and thiocyanate in cord serum; Acta Obstet. Gynecol. Scand. 75 (1996) 902-907.

167. Rosevear, S.K., D.W. Holt, T.D. Lee, W.C.L. Ford, P.G. Wardle, and M.G.R. Hull: Smoking and decreased fertilization rates in vitro; Lancet 340 (1992) 1195-1196.

168. Zenzes, M.T., T.E. Reed, P. Wang, and J. Klein: Cotinine, a major metabolite of nicotine, is detectable in follicular fluids of passive smokers in in vitro fertilization therapy; Fertil. Steril. 66 (1996) 614-619.

169. Younglai, E.V., W.G. Foster, E.G. Hughes, K. Trim, and J.F. Jarrell: Levels of environmental contaminants in human follicular fluid, serum and seminal plasma of couples undergoing in vitro fertilization; Arch. Environ. Contam. Toxicol. 43 (2002) 121-126.

170. Knight, J.M., C. Eliopoulos, J. Klein, M. Greenwald, and G. Koren: Passive smoking in children. Racial differences in systemic exposure to cotinine by hair and urine analysis; Chest 109 (1996) 446-450.

171.Zenzes, M.T., R. Bielecki, and T.E. Reed: Detection of benzo(a)pyrene diol epoxide-DNA adducts in sperm of men exposed to cigarette smoke; Fertil. Steril. 72 (1999) 330-335.

172. Wong, W.Y., C.M.G. Thomas, H.M.W.M. Merkus, G.A. Zielhuis, W.H. Doesburg, and R.P.M. SteegersTheunissen: Cigarette smoking and the risk of male factor subfertility: minor association between cotinine in seminal plasma and semen morphology; Fertil. Steril. 74 (2000) 900-935.

173. Van Vunakis, H., H.B. Gijka, and J.J. Langone: Radioimmunoassay for nicotine and cotinine; in: Environmental carcinogens-methods of analysis and exposure measurement, Vol. 9, International Agency for Research on Cancer, edited by I.K. O'Neill, K.D. Brunnemann, B. Bodet, and D. Hoffmann, Lyon, 1987, pp. 317-330.

174. Watts, R.R., J.J. Langone, G.J. Knight, and J. Lewtas: Cotinine analytical workshop report: consideration of analytical methods for determining cotinine in human body fluids as a measure of passive exposure to tobacco smoke; Environ. Health Perspect. 84 (1990) 173-182.

175. Schepers, G. and R.-A. Walk: Cotinine determination by immunoassays may be influenced by other nicotine metabolites; Arch. Toxicol. 62 (1988) 395-397.

176. Zuccaro, P., S. Pichini, I. Altieri, M. Rosa, M. Pellegrini, and R. Pacifici: Interference of nicotine metabolites in cotinine determination by RIA; Clin. Chem. 43 (1997) 181-182.

177. Hansen, A.M., A.H. Garde, J.M. Christensen, N. Eller, L.E. Knudsen, and R. Heinrich-Ramm: Reference interval and subject variation in excretion of urinary metabolites of nicotine from non-smoking healthy subjects in Denmark; Clin. Chim. Acta 304 (2001) 125-132.

178. Fergusson, B.B., D.J. Wilson, and W. Schaffner: Determination of nicotine concentrations in human milk; Am. J. Dis. Child. 130 (1976) 837-839.

179. Hengen, N. and M. Hengen: Gas-liquid chromato- 
graphic determination of nicotine and cotinine in plasma; Clin. Chem. 24 (1978) 50-53.

180. Feyerabend, C. and M.A.H. Russell: Improved gaschromatographic method and micro-extraction technique for the measurement of nicotine in biological fluids; J. Pharm. Pharmacol. 31 (1979) 73-76.

181. Jacob, P., III., M. Wilson, and N.L. Benowitz: Improved gas chromatographic method for the determination of nicotine and cotinine in biologic fluids; $\mathrm{J}$. Chromatogr. 222 (1981) 61-70.

182. Jacob, P., III.,A.T. Shulgin, L. Yu, and N.L. Benowitz: Determination of the nicotine metabolite trans-3'hydroxycotinine in urine of smokers using gas chromatography with nitrogen-selective detection or selected ion monitoring; J. Chromatogr. 583 (1992) 145-154.

183. Kogan, M.J., K. Verebey, J.H. Jaffee, and S.J. Mulé: Simultaneous determination of nicotine and cotinine in human plasma by nitrogen detection gas-liquid chromatography; J. Forensic Sci. 26 (1981) 6-11.

184. Stehlik, G., J. Kainzbauer, H. Tausch, and O. Richter: Improved method for routine determination of nicotine and its main metabolites in biological fluids; J. Chromatogr. 232 (1982) 295-303.

185. Davis, R.A.: The determination of nicotine and cotinine in plasma; J. Chrom. Sci. 24 (1986) 134-141.

186. Voncken, P., G. Schepers, and K.H. Schäfer: Capillary gas chromatographic determination of trans-3'-hydroxycotinine simultaneously with nicotine and cotinine in urine and blood samples; J. Chromatogr. 479 (1989) 410-418.

187. Petrakis, N.L., L.D. Gruenke, T.C. Beelen, N. Castagnoli, and J.C. Craig: Nicotine in breast fluid of nonlactating women; Science 199 (1978) 303-304.

188. Davoli, E., L. Stramare, R. Fanelli, L. Diomede, and M. Salmona: Rapid solid-phase extraction method for automated gas chromatographic-mass spectrometric determination of nicotine in plasma; J. Chromatogr. B 707 (1998) 312-316.

189. Daenens, P., L. Laruelle, K. Callewaert, P. De Schepper, R. Galeazzi, and J. van Rossum: Determination of cotinine in biological fluids by capillary gas chromatography-mass spectrometry-selected-ion monitoring; J. Chromatogr. 342 (1985) 79-87.

190. Skarping, G., S. Willers, and M. Dalene: Determination of the cotinine in urine using glass capillary gas chromatography and selective detection, with special reference to the biological monitoring of passive smoking; J. Chromatogr. 454 (1988) 293-301.

191. McAdams, S.A. and M.L. Cordeiro: Simple selected ion monitoring capillary gas chromatographic-mass spectrometric method for the determination of cotinine in serum, urine and oral samples; J. Chromatogr. 615 (1993) 148-153.

192. Lewis, S.J., N.M. Cherry, R.M.C.L. Niven, P.V. Barber, K. Wilde, and A.C. Povey: Cotinine levels and self-reported smoking status in patients attending a bronchoscopy clinic; Biomarkers 8 (2003) 218-228.

193. James, H., Y. Tizabi, and R. Taylor: Rapid method for the simultaneous measurement of nicotine and cotinine in urine and serum by gas chromatography-mass spectrometry; J. Chromatogr. B 708 (1998) 87-93.

194. Shin, H.S., J.G. Kim, Y.J. Shin, and S.H. Jee: Sensitive and simple method for the determination of nicotine and cotinine in human urine, plasma and saliva by gas chromatography - mass spectrometry; J. Chromatogr. B
769 (2002) 177-183.

195. Cognard, E. and C. Staub: Determination of nicotine and its major metabolite cotinine in plasma or serum by gas chromatography-mass spectrometry using ion-trap detection; Clin. Chem. Lab. Med. 41 (2003) 1599-1607.

196. Torańo, J.S. and H.J.M. van Kan: Simultaneous determination of the tobacco smoke uptake parameters nicotine, cotinine and thiocyanate in urine, saliva and hair, using gas chromatography-mass spectrometry for characterisation of smoking status of recently exposed subjects; Analyst 128 (2003) 838-843.

197. Mousa, S., G.R. Van Loon, A.A. Houdi, and P.A. Crooks: High-performance liquid chromatography with electrochemical detection for the determination of nicotine and $\mathrm{N}$-methylnicotinium ion; J. Chromatogr. 347 (1985) 405-410.

198. Chien, C., J.N. Diana, and P.A. Crooks, High-performance liquid chromatography with electrochemical detection for the determination of nicotine in plasma; J. Pharm. Sci. 77 (1988) 277-279.

199. Mahoney, G.N. and W. Al-Delaimy: Measurement of nicotine in hair by reversed-phase high-performance liquid chromatography with electrochemical detection; J. Chromatogr. 753 (2001) 179-187.

200. Machacek, D.A. and N.-S. Jiang: Quantification of cotinine in plasma and saliva by liquid chromatography; Clin. Chem. 32 (1986) 979-982.

201. Shen, H., S. Chia, Z. Ni, A. New, B. Lee, and C. Ong: Detection of oxidative damage in human sperm and the association with cigarette smoking; Reprod. Toxicol. 11 (1997) 675-680.

202. Greaves, R., L. Trotter, S. Brennecke, and E. Janus: A simple high-pressure liquid chromatography cotinine assay: validation of smoking status in pregnant women; Ann. Clin. Biochem. 38 (2001) 333-338.

203. Baranowski, J., G. Pochopień, and I. Baranowska: Determination of nicotine, cotinine and caffeine in meconium using high-performance liquid chromatography; J. Chromatogr. B 707 (1998) 317-321.

204. Harlharan, M., T. VanNoord, and J.F. Greden: A highperformance liquid chromatographic method for routine simultaneous determination of nicotine and cotinine in plasma; Clin. Chem. 34 (1988) 724-729.

205. Page-Sharp, M., T.W. Hale, L.P. Hackett, J.H. Kristensen, and K.F. Ilett: Measurement of nicotine and cotinine in human milk by high-performance liquid chromatography with ultraviolet absorbance detection; J. Chromatogr. B 796 (2003) 173-180.

206. Ghosheh, O.H., D. Browne, T. Rogers, J. de Leon, L.P. Dwoskin, and P.A. Crooks: A simple high performance liquid chromatographic method for the quantification of total cotinine, total 3'-hydroxycotinine and caffeine in the plasma of smokers; J. Pharm. Biomed. Anal. 23 (2000) 543-549.

207. Cundy, K.C. and P.A. Crooks: High-performance liquid chromatographic method for the determination of $\mathrm{N}$ methylated metabolites of nicotine; J. Chromatogr. 306 (1984) 291-301.

208. Kyerematen, G.A., L.H. Taylor, J.D. deBethizy, and E.S. Vesell: Radiometric-high-performance liquid chromatographic assay for nicotine and twelve of its metabolites; J. Chromatogr. 419 (1987) 191-203.

209. Pacifici, R., I. Altiere, L. Gandini, A. Lenzi, S. Pichini, M. Rosa, P. Zuccaro, and F. Dondero: Nicotine, cotinine and trans-3'-hydroxycotinine levels in seminal plasma of smokers: effects on sperm parameters; Ther. 
Drug Monit. 15 (1993) 358-363.

210. McManus, K.T., J.D. deBethizy, D.A. Garteiz, G.A. Kyerematen, and E.S. Vesell: A new quantitative thermospray LC-MS method for nicotine and its metabolites in biological fluids; J. Chrom. Sci. 28 (1990) 510-516.

211. Byrd, G.D., M.S. Uhrig, J.D. deBethizy, W.S. Caldwell, P.A. Crooks, A. Ravard, and R.M. Riggs: Direct determination of cotinine- $N$-glucuronide in urine using thermospray liquid chromatography/mass spectrometry; Biol. Mass Spectrom. 23 (1994) 103-107.

212. Bentley, M.C., M. Abrar, M. Kelk, J. Cook, and K. Phillips: Validation of an assay for the determination of cotinine and 3-hydroxycotinine in human saliva using automated solid-phase extraction and liquid chromatography with tandem mass spectrometry detection; J. Chromatogr. 723 (1999) 185-194.

213. Byrd, G.D., R.A. Davis, and M.W. Ogden: A rapid LCMS-MS method for the determination of nicotine and cotinine in serum and saliva samples from smokers: Validation and comparison with a radioimmunoassay method; J. Chrom. Sci. 43 (2005) 133-140.

214. Bernert, J.T., Jr., J.E. McGuffey, M.A. Morrison, and J.L. Pirkle: Comparison of serum and salivary cotinine measurements by a sensitive high-performance liquid chromatography-tandem mass spectrometry method as an indicator of exposure to tobacco smoke among smokers and nonsmokers; J. Anal. Toxicol. 24 (2000) 333-339.

215. Bernert, J.T., W.E. Turner, J.L. Pirkle, C.S. Sosnoff, J.R. Akins, M.K. Waldrep, Q. Ann, T.R. Covey, W.E. Whitfield, E.W. Gunter, B.B. Miller, D.G. Patterson, Jr., L.L. Needham, W.H. Hannon, and E.J. Sampson: Development and validation of sensitive method for determination of serum cotinine in smokers and nonsmokers by liquid chromatography/atmospheric pressure ionization tandem mass spectrometry; Clin. Chem. 43 (1997) 2281-2291.

216. Kellogg, M.D., J. Behaderovic, O. Bhalala, and N. Rifai: Rapid and simple tandem mass spectrometry method for determination of serum cotinine concentration; Clin. Chem. 50 (2004) 2157-2159.

217. Xu, A.S., L.L. Peng, J.A. Havel, M.E. Peterson, J.A. Fiene, and J.D. Hulse: Determination of nicotine and cotinine in human plasma by liquid chromatographytandem mass spectrometry with atmospheric pressure chemical ionization interface; J. Chromatogr. B. 682 (1996) 249-257.

218. Taylor, P.J., K.K. Forrest, P.G. Landsberg, C. Mitchell, and P.I. Pillans: The measurement of nicotine in human plasma by high-performance liquid chromatographyelectrospray-tandem mass spectrometry; Ther. Drug Monit. 26 (2004) 563-568.

219. Moyer, T.P., J.R. Charlson, R.J. Enger, L.C. Dale, J.O. Ebbert, D.R. Schroeder, and R.D. Hurt: Simultaneous analysis of nicotine, nicotine metabolites and tobacco alkaloids in serum or urine by tandem mass spectrometry, with clinically relevant metabolic profiles; Clin. Chem. 48 (2002) 1460-1471.

220. Xu, X., M.M. Iba, and C.P. Weisel: Simultaneous and sensitive measurement of anabasine, nicotine and nicotine metabolites in human urine by liquid chromatography-tandem mass spectrometry; Clin. Chem. 50 (2004) 2323-2330.

221. Yamanaka, H., M. Nakajima, K. Nishimura, R. Yoshida, T. Fukami, M. Katoh, and T. Yokoi: Meta- bolic profile of nicotine in subjects whose CYP2A6 gene is deleted; Eur. J. Pharm. Sci. 22 (2004) 419-425.

222. Heavner, D.L., J.D. Richardson, W.T. Morgan, and M.W. Ogden: Validation and application of a method for the determination of nicotine and five major metabolites in smokers' urine by solid-phase extraction and liquid chromatography-tandem mass spectrometry; Biomed. Chromatogr. 19 (2005) 312-328.

223. Karnes, H.T., J.R. James, C. March, D.E. Leyden, and K. Koller: Assessment of nicotine uptake from cigarette smoke: comparison of a colorimetric test strip (NicCheck $\mathrm{I}^{\mathrm{TM}}$ ) and gas chromatography/mass selective detector; Biomarkers 6 (2001) 388-399.

224. Gariti, P., D.I. Rosenthal, K. Lindell, J. Hansen-Flaschen, J. Shrager, C. Lipkin, A.I. Alterman, and L.R. Kaiser: Validating a dipstick method for detecting recent smoking; Cancer Epidemiol. Biomarkers Prev. 11 (2002) 1123-1125.

225. Parker, D.R., T.M. Lasater, R. Windsor, J. Wilkins, D.I. Upegui, and J. Heimdal: The accuracy of self-reported smoking status assessed by cotinine test strips; Nicotine Tob. Res. 4 (2002) 305-309.

226. Berry, D.J. and J. Grove: Inproved chromatographic techniques and their interpretation for the screening of urine from drug dependent subjects; J. Chromatogr. 61 (1971) 111-116.

227. Bazylak, G., H. Brózik, and W. Sabanty: Combined SPE and HPTLC as a screening assay of urinary cotinine from male adolescents exposed to environmental tobacco smoke; Polish J. Environ. Studies 9 (2000) 113-123.

228. Palmer, M.E., R.F. Smith, K. Chamber, and L.W. Tetler: Separation of nicotine metabolites by capillary zone electrophoresis and capillary zone electrophores/mass spectrometry; Rapid Comm. Mass Spectrom. 15 (2001) 224-231.

229. Baidoo, E.E.K., M.R. Clench, R.F. Smith, and L.W. Tetler: Determination of nicotine and its metabolites in urine by solid-phase extraction and sample stacking capillary electrophoresis-mass spectrometry; J. Chromatogr. B 796 (2003) 303-313.

230. Jarvis, M.J. and M.A.H. Russell: Passive exposure to tobacco smoke; Br. Med. J. 291 (1985) 1646.

231. Curvall, M., E. Kazemi-Vala, J. Wahren, and C. Enzell: Concentration of cotinine body fluids as a measure of nicotine intake by nonsmokers; in: The Pharmacology of Nicotine, edited by M.J. Rand and K. Thurau, IRL Press, Oxford, 1988, pp. 32-33.

232. Jarvis, M.J., P. Primatesta, B. Erens, C. Feyerabend, and A. Bryant: Measuring nicotine intake in population surveys: comparability of saliva cotinine and plasma cotinine estimates; Nicotine Tob. Res. 5 (2003) 349-355.

233. Wewers, M.E., K.L. Ahijevych, R.K. Dhatt, R.M. Guthrie, P. Kuun, L. Mitchell, M.L. Moeschberger, and M.S. Chen Jr.: Cotinine levels in Southeast Asian smokers; Nicotine Tob. Res. 2 (2000) 85-91.

234. Jarvis, M., H. Tunstall-Pedoe, C. Feyerabend, C. Vesey, and Y. Salloojee: Biochemical markers of smoke absorption and self reported exposure to passive smoking; J. Epidemiol. Commun. Health 38 (1984) 335-339.

235. Jarvis, M.J., M.A.H. Russell, N.L. Benowitz, and C. Feyerabend: Elimination of cotinine from body fluids: implications for noninvasive measurement of tobacco smoke exposure; Am. J. Public Health 78 (1988) 696-698. 
236. Wall, M.A., J. Johnson, P. Jacob, and N.L. Benowitz: Cotinine in the serum, saliva and urine of nonsmokers, passive smokers and active smokers; Am. J. Public Health 78 (1988) 699-701.

237. Coultas, D.B., J.M. Samet, J.F. McCarthy, and J.D. Spengler: Variability of measures of exposure to environmental tobacco smoke in the home; Am. Rev. Respir. Dis. 142 (1990) 602-606.

238. Berlin, I., A. Radzius, J.E. Henningfield, and E.T. Moolchan: Correlates of expired air carbon monoxide: effect of ethnicity and relationship with saliva cotinine and nicotine; Nicotine Tob. Res. 3 (2001) 325-331.

239. Dolcini, M.M., N.E. Adler, P. Lee, and K.E. Bauman: An assessment of the validity of adolescent selfreported smoking status using three biological markers; Nicotine Tob. Res. 5 (2003) 473-383.

240. Stookey, G.K., B.P. Katz, B.L. Olsen, C.A. Drook, and S.J. Cohen: Evaluation of biochemical validation measures in determination of smoking status; J. Dent. Res. 66 (1987) 1597-1601.

241. Metz, C.E.: Basic principles of ROC analysis. Seminars Nuclear Med. 8 (1978) 283-298.

242. Cummings, S.R. and R.J. Richard: Optimum cutoff points for biochemical validation of smoking status; Am. J. Public Health 78 (1988) 574-575.

243. Wagenknecht, L.E., G.L. Burke, L.L. Perkins, N.J. Haley, and G.D. Friedman: Misclassification of smoking status in the CARDIA study: a comparison of self-report with serum cotinine levels; Am. J. Public Health 82 (1992) 33-36.

244. Caraballo, R.S., G.A. Giovino; and T.F. Pechacek: Selfreported cigarette smoking vs. serum cotinine among U.S. adolescents; Nicotine Tob. Res. 6 (2004) 19-25.

245. McNeill, A.D., M.J. Jarvis, R. West, M.A.H. Russell, and A. Bryant: Saliva cotinine as an indicator of cigarette smoking in adolescents; Br. J. Addict. 82 (1987) 1355-1360.

246. Wagenknecht, L.E., G.R. Cutter, N.J. Haley, S. Sidney, T.A. Manolio, G.H. Hughes, and D.R. Jacob: Racial differences in serum cotinine levels among smokers in the Coronary Artery Risk Development in (Young) Adults Study; Am. J. Public Health 80 (1990) 1053-1056.

247. English, P.B., B. Eskenazi, and R.E. Christianson: Black-white differences in serum cotinine levels among pregnant women and subsequent effects on infant birthweight; Am. J. Public Health 84 (1994) 1439-1443.

248. Pérez-Stable, E.J., G. Marín, B.V. Marín, and N.L. Benowitz: Misclassification of smoking status by selfreported cigarette consumption; Am. Rev. Resp. Dis. 145 (1992) 53-57.

249. Caraballo, R.S., G.A. Giovino, T.F. Pechacek, and P.D. Mowery: Factors associated with discrepancies between self-reports on cigarette smoking and measured serum cotinine levels among persons aged 17 years or older; Am. J. Epidemiol. 153 (2001) 807-814.

250. Owen, L. and A. McNeill: Saliva cotinine as indicator of cigarette smoking in pregnant women; Addiction 96 (2001) 1001-1006.

251. Jacob, P., III., L. Yu, A.T. Shulgin, and N.L. Benowitz: Minor tobacco alkaloids as biomarkers for tobacco use: comparison of users of cigarette, smokeless tobacco, cigar and pipes; Am. J. Public Health 89 (1999) 731-736.

252. Jacob, P., III., D. Hatsukami, H. Severson, S. Hall, L. $\mathrm{Yu}$, and N.L. Benowitz: Anabasine and anatabine as biomarkers for tobacco use during nicotine replacement therapy; Cancer Epidemiol. Biomarker Prev. 11 (2002) 1668-1673.

253. Feyerabend, C. and M.A.H. Russell: Effect of urinary $\mathrm{pH}$ and nicotine excretion rate on plasma nicotine during cigarette smoking and chewing nicotine gum; Br. J. clin. Pharmac. 5 (1978) 293-297.

254. Benowitz, N.L., P. Jacob, III., R.T. Jones, and J. Rosenberg: Interindividual variability in the metabolism and cardiovascular effects of nicotine in man; J. Pharmacol. Exp. Ther. 221 (1982) 368-372.

255. Benowitz, N.L., F. Kuyt, P. Jacob, III., R.T. Jones, and A.-L. Osman: Cotinine disposition and effects; Clin. Pharmacol. Ther. 34 (1983) 604-611.

256. Isaac, P.F. and M.J. Rand, Cigarette smoking and plasma levels of nicotine; Nature 236 (1972) 308-310.

257. Foulds, J., A. Bryant, J. Stapleton, M.J. Jarvis, and M.A.H. Russell: The stability of cotinine in unfrozen saliva mailed to the laboratory; Am. J. Public Health 81 (1994) 1182-1183.

258. Vartiainen, E., T. Seppälä, P. Lillsunde, and P. Puska: Validation of self reported smoking by serum cotinine measurement in a community-based study; J. Epidemiol. Community Health 56 (2000) 167-170.

259. Pérez-Stable, E.J., N.L. Benowitz, and G. Marín: Is serum cotinine a better measure of cigarette smoking than self-report?; Prev. Med. 24 (1995) 171-179.

260. Benowitz, N.L. and D.S. Sharp: Inverse relation between serum cotinine concentration and blood pressure in cigarette smokers; Circulation 80 (1989) 1309-1312.

261. Caraballo, R.S., G.A. Giovino, T.F. Pechacek, P.D. Mowery, P.A. Richter, W.J. Strauss, D.J. Sharp, M.P. Eriksen, J.L. Pirkle, and K.R. Maurer: Racial and ethnic differences in serum cotinine levels of cigarette smokers; JAMA 280 (1998) 135-139.

262. Wagenknecht, L.E., T.A. Manolio, S. Sidney, G.L. Burke, and N.J. Haley: Environmental tobacco smoke exposure as determined by cotinine in black and white young adults: the CARDIA Study; Environ. Res. 63 (1993) 39-46.

263. Crawford, F.G., J. Mayer, R.M. Santella, T.B. Cooper, R. Ottman, W.Y. Tsai, G. Simon-Cereijido, M. Wang, D. Tang, and F.P. Perera: Biomarkers of environmental tobacco smoke in pre-school children and their mothers; J. Natl. Cancer Inst. 86 (1994) 1398-1402.

264. Pirkle, J.L., K.M. Flegal, J.T. Bernert, D.J. Brody, R.A. Etzel, and K.R. Maurer: Exposure of the US population to environmental tobacco smoke. The Third National Health and Nutrition Examination Survey 1988 to 1991; JAMA 275 (1996) 1233-1240.

265. Jarvis, M.J., C. Feyerabend, A. Bryant, B. Hedges, and P. Primatesta: Passive smoking in the home: plasma cotinine concentrations in non-smokers with smoking partners; Tobacco Control 10 (2001) 368-374.

266. Kaufman, F.L., M. Kharrazi, G.N. DeLorenze, B. Eskenazi, and J.T. Bernert: Estimation of environmental tobacco smoke exposure during pregnancy using a single question on household smokers verses serum cotinine; J. Exp. Anal. Environ. Epidemiol. 12 (2002) 286-295.

267. Pattishall, E.N., G.L. Strope, R.A. Etzel, R.W. Helms, N.J. Haley, and F.W. Denny: Serum cotinine as a measure of tobacco smoke exposure in children; Am. J. Dis. Child. 139 (1985) 1101-1104.

268. Mannino, D.M., R. Caraballo, N. Benowitz, and J. 
Repace: Predictors of cotinine levels in US children. Data from the Third National Health and Nutrition Examination Survey; Chest 120 (2001) 718-724.

269. Mercelina-Roumans, P.E.A.M., H. Schouten, J.M.H. Ubachs, and J.W.J. van Wersch: Cotinine concentrations in plasma of smoking pregnant women and their infants; Eur. J. Clin. Chem. Biochem. 34 (1996) 525-528.

270. Pichini, S., X. Basagana, R. Pacifici, O. Garcia, C. Puig, O. Vall, J. Harris, P. Zuccaro, J. Segura, and J. Sunyer: Cord serum cotinine as a biomarker of fetal exposure to cigarette smoke at the end of pregnancy; Env. Health Perspect. 108 (2000) 1079-1083.

271.Pojer, R., J.B. Whitfield, V. Poulos, I.F. Eckhard, R. Richmond, and W.J. Hensley: Carboxyhemoglobin, cotinine and thiocyanate assay compared for distinguishing smokers from non-smokers; Clin. Chem. 30 (1984) 1377-1380.

272. de Leon, J., F.J. Diaz, T. Rogers, D. Browne, L. Dinsmore, O.H. Gosheh, L.P. Dwoskin, and P.A. Crooks: Plasma cotinine 3'-hydroxycotinine and their glucuronides in white and black smokers; J. Clin. Psychopharmacol. 23 (2003) 209-211.

273. Galeazzi, R.L., P. Daenens, and M. Gugger: Steadystate concentration of cotinine as a measure of nicotineintake by smokers; Eur. J. Clin. Pharmacol. 28 (1985) 301-304.

274. Ashton, H., R. Stepney, and J.W. Thompson: Selftitration by cigarette smokers; Br. Med. J. 2 (1979) 357-360.

275.Zacny, J.P., M.L. Stitzer, F.J. Brown, J.E. Yingling, and R.R. Griffiths: Human cigarette smoking: effects of puff and inhalation parameters on smoke exposure; J. Pharmacol. Expt. Ther. 240 (1987) 554-564.

276. Hatsumaki, D.K., R.W. Pickens, D.S. Svikis, and J.R. Hughes: Smoking topography and nicotine blood levels; Addict. Behav. 13 (1988) 91-95.

277. Höfer, I., R. Nil, and K. Bättig: Nicotine yield as determinant of smoke exposure indicators and puffing behavior; Pharmacol. Biochem. Behav. 40 (1991) 139-149.

278. Patterson, F., N. Benowitz, P. Shields, V. Kaufmann, C. Jepson, P. Wileyto, S. Kucharski, and C. Lerman: Individual differences in nicotine intake per cigarette; Cancer Epidemiol; Biomarkers Prev. 12 (2003) 468-471.

279. Malson, J.L., E.M. Lee, R. Murty, E.T. Moolchan, and W.B. Pickworth: Clove cigarette smoking: biochemical, physiological and subjective effects; Pharmacol. Biochem. Behav. 74 (2003) 739-745.

280. Baker, R.R. and M. Dixon: The retention of tobacco smoke constituents in the human respiratory tract; Inhalation Toxicol. 17 (2006) 1-39.

281. Olivieri, M., A. Poli, P. Zuccaro, M. Ferrari, G. Lampronti, R. de Marco, V.L. Cascio, and R. Pacifici: Tobacco smoke exposure and serum cotinine in a random sample of adults living in Verona, Italy; Arch. Environ. Health 57 (2002) 355-359.

282. Rosa, M., R. Pacifici, I. Altieri, S. Pichini, G. Ottaviani, and P. Zuccaro: How the steady-state cotinine concentration in cigarette smokers is directly related to nicotine intake; Clin. Pharmacol. Ther. 52 (1992) 324-329.

283. SRNT Subcommittee on Biochemical Verification: Biochemical verification of tobacco use and cessation; Nicotine Tob. Res. 4 (2002) 149-159.

284. US Public Health Service: Healthy People 2010: National Health Promotion and Disease Prevention
Objectives [full report, with commentary]; Department of Health and Human Services, Washington DC, 2000.

285. Assaf, A.R., D. Parker, K.L. Lapane, J.L. McKenney, and R.A. Carleton: Are gender differences in selfreported smoking practices? Correlation with thiocyanate and cotinine levels in smokers and nonsmokers from the Pawtucket Heart Health Program; J. Women's Health 11 (2002) 899-906.

286. A Report of the Surgeon General: Tobacco Use Among U.S. Racial/Ethnic Minoroty Groups - African Americans, American Indians and Alaska Natives, Asian Americans and Pacific Islanders and Hispanics; Rockville, Maryland, US Department of Health and Human Services, 1989.

287. de Leon, J., F.J. Diaz, T. Rogers, D. Browne, L. Dinsmore, O.H. Gosheh, L.P. Dwoskin, and P.A. Crooks: Total cotinine in plasma: a stable biomarker for exposure to tobacco smoke; J. Clin. Psychopharmacol. 22 (2002) 496-501.

288. Lambers, D.S. and K.E. Clark: The maternal and fetal physiologic effects of nicotine; Semin. Perinatol. 20 (1996) 115-126.

289. Bearer, C., R.K. Emerson, M.A. O'Riordan, E. Roitman, and C. Shackleton: Maternal tobacco smoke exposure and persistent pulmonary hypertension of the newborn; Environ. Health Perspect. 105 (1997) 202-206.

290. Benowitz, N.L.: Biomarkers of environmental tobacco smoke exposure; Environ. Health Perspect. 107 (1999) 349-355.

291. Feyerabend, C., T. Higenbottam, and M.A.H. Russell: Nicotine concentrations in urine and saliva of smokers and non-smokers; Br. Med. J. 284 (1982) 1002-1004.

292. Abrams, D.B., M.J. Follick, L. Biener, K.B. Carey, and J. Hitti: Saliva cotinine as a measure of smoking status in field settings; Am. J. Public Health 77 (1987) 846-848.

293.Lee, P.N.: Lung cancer and passive smoking: association an artefact due to missclasification of smoking habits?; Toxicol. Lett. 35 (1987) 157-162.

294.Etter, J.-F., T.V. Perneger, and A. Ronchi: Collecting saliva samples by mail; Am. J. Epidemiol. 147 (1998) 141-146.

295.Etter, J.-F., T. Vu Duc, and T.V. Perneger: Saliva cotinine levels in smokers and nonsmokers; Am. J. Epidemiol. 151 (2000) 251-258.

296. Schneider, N.G., P. Jacob III., F. Nilsson, S.J. Leischow, N.L. Benowitz, and R.E. Olmstead: Saliva cotinine levels as a function of collection method; Addiction 92 (1997) 347-351.

297. Feyerabend, C. and M.A.H. Russell: A rapid gasliquid chromatographic method for the determination of cotinine and nicotine in biological fluids; J. Pharm. Pharmacol. 42 (1990) 450-452.

298. Delifino, R.J., P. Ernst, M.S. Jaakkola, S. Solomon, and M.R. Becklake: Questionnaire assessments of recent exposure to environmental tobacco smoke in relation to salivary cotinine; Eur. Respir. J. 6 (1993) 1104-1108.

299. Jarvis, M.J., E. Goddard, V. Higgins, C. Feyerabend, A. Bryant, and D.G. Cook: Children's exposure to passive smoking in England since the 1980s: cotinine evidence from population surveys; Br. Med. J. 321 (2000) 343-345.

300. Pierce, J.P., T. Dwyer, E. DiGiusto, T. Carpenter, C. Hannam, A. Amin, C. Yong, G. Sarfaty, J. Shaw, and N. Burke: Cotinine validation of self-reported smoking 
in commercially run community surveys; J. Chronic Dis. 40 (1987) 689-695.

301. Etzel, R.A.: A review of the use of saliva cotinine as a marker of tobacco smoke exposure; Prev. Med. 19 (1990) 190-197.

302. Cummings, K.M., S.J. Markello, M. Mahoney, A.K. Bhargava, P.D. McElroy, and J.R. Marshall: Measurement of current exposure to environmental tobacco smoke; Arch. Environ. Health 45 (1990) 74-79.

303. Beckett, A.H., M. Rowland, and E.J. Triggs: Significance of smoking in investigations of urinary excretion rates of amines in man; Nature 207 (1965) 200-201.

304. Labrecque, M., S. Marcoux, J.-P. Weber, J. Fabia, and L. Ferron: Feeding and urine cotinine values in babies whose mothers smoke; Pediatrics 83 (1989) 93-97.

305. Thompson, S.G., R.D. Barlow, N.J. Wald, and H. Van Vunakis: How should urinary cotinine concentrations be adjusted for urinary creatinine concentration? Clin. Chim. Acta 187 (1990) 289-295.

306. Dahlström, A., B. Lundell, M. Curvall, and L. Thapper: Nicotine and cotinine concentrations in the nursing mother and her infant; Acta Paediatr. Scand. 79 (1990) 142-147.

307. Köhler, E., D. Bretschneider, A. Rabsilber, W. Weise, and G. Jorch: Assessment of prenatal smoke exposure by determining nicotine and its metabolites in maternal and neonatal urine; Human Exp. Toxicol. 20 (2001) 1-7.

308. Cornelius, M.D., L. Goldschmidt, and D.A. Dempsey: Environmental tobacco smoke exposure in lowincome 6-year-olds: Parent report and urine cotinine measures; Nicotine Tob. Res. 5 (2003) 333-339.

309. Haddow, J.E., G.J. Knight, G.E. Palomaki, L.M. Neveux, and B.A. Chilmonczyk: Replacing creatinine measurements with specific gravity values to adjust urine cotinine concentrations; Clin. Chem. 40 (1994) 562-564.

310. Heavner, D.L., W.T. Morgan, S.B. Sears, J.D. Richardson, G.D. Byrd, and M.W. Ogden: Effect of creatinine and specific agravity normalization techniques on xenobiotic biomarkers in smokers' spot and 24-h urines; J. Pharm. Biomed. Anal. 40 (2006) 928-942.

311. Jatlow, P., S. McKee, and S.S. O'Malley: Correction of urine cotinine concentrations for creatinine excretion: is it useful? Clin. Chem. 49 (2003) 1932-1934.

312. Luck, W. and H. Nau: Nicotine and cotinine concentrations in serum and milk of nursing smokers; Br. J. clin. Pharmac. 18 (1984) 9-15.

313. Hagan, R.L., J.M. Ramos Jr., and P.M. Jacob III.: Increasing urinary cotinine concentrations at elevated temperatures: the role of conjugated metabolites; J. Pharmaceutical Biomedical Anal. 16 (1997) 191-197.

314. Riboli, E., N.J. Haley., F. De Waard, and R. Saracci: Validity of urinary biomarkers of exposure to tobacco smoke following prolonged storage; Int. J. Epidemiol. 24 (1995) 354-358.

315. Jacqz-Aigrain, E., D. Zhang, G. Maillard, D. Luton, J. André, and J.F. Oury: Maternal smoking during pregnancy and nicotine and cotinine concentrations in maternal and neonatal hair; Br. J. Obstet. Gynaecol. 109 (2002) 909-911.

316. Riboli, E., N.J. Haley., J. Trédaniel, R. Saracci, S. Preston-Martin, and D. Trichopoulos: Misclassification of smoking status among women in relation to exposure to environmental tobacco smoke; Eur. Respir. J. 8
(1995) 285-290.

317. Nafstad, P., G. Botten, J.A. Hagen, K. Zahlsen, O.G., Nilsen, T. Silsand, and J. Kongerud: Comparison of three methods for estimating environmental tobacco smoke exposure among children aged between 12 and 36 months; Int. J. Epidemiol. 24 (1995) 88-94.

318. Matt, G.E., D.R. Wahlgren, M.F. Hovell, J.M. Zakarian, J.T. Bernert, S.B. Meltzer, J.L. Pirkle, and S. Caudill: Measuring environmental tobacco smoke exposure in infants and young children through urine cotinine and memory-based parental reports: empirical findings and discussion; Tobacco Control 8 (1999) 282-289.

319. Luck, W. and H. Nau: Nicotine and cotinine concentrations in serum and urine of infants exposed via passive smoking or milk from smoking mothers; J. Pediatr. 107 (1985) 816-820.

320. Woodward, A., N. Grgurinovich, and P. Ryan: Breast feeding and smoking hygiene: major influences on cotinine in urine of smokers' infants; J. Epidemiol. Community Health 40 (1986) 309-315.

321. Thompson, S.G., R. Stone, K. Nanchahal, and N.J. Wald: Relation of urinary cotinine concentrations to cigarette smoking and to exposure to other people's smoke; Thorax 45 (1990) 356-361.

322. Boswell, C., M. Curvall, R.K. Elswick Jr. and D. Leyden: Modelling nicotine intake in smokers and snuff users using biological fluid nicotine metabolites; Biomarkers 5 (2000) 341-354.

323. Schepers, G. and D. Demetriou: Determination of nicotine and its metabolites in urine by HPLC after DETBA derivatization; $8^{\text {th }}$ Annual Society for Research and Tobacco Conference 20-23 ${ }^{\text {rd }}$ February 2002, Savannah, Georgia, US.

324. Schulte-Hobein, B., D. Schwartz-Bickenbach, S. Abt, C. Plum, and H. Nau: Cigarette smoke exposure and development of infants throughout the first year of life: influence of passive smoking and nursing on cotinine levels in breast milk and infant's urine; Acta Paediatr. 81 (1992) 550-557.

325. Schwartz-Bickenbach, D., B. Schulte-Hobein, S. Abt, C. Plum, and H. Nau: Smoking and passive smoking during pregnancy and early infancy: effects on birth weight, lactation period and cotinine concentrations in mother's milk and infant's urine; Toxicol. Lett. 35 (1987) 73-81.

326. Kintz, P. and P. Mangin: Determination of gestational opiate, nicotine, benzodiazepine, cocaine and amphetamine exposure by hair analysis; J. Forensic Sci. Soc. 33 (1993) 139-142.

327. Uematsu, T.: Therapeutic drug monitoring in hair samples; Clin. Pharmacokinet. 25 (1993) 83-87.

328. Al-Delaimy, W.K.: Hair as a biomarker for exposure to tobacco smoke; Tobacco Control 11 (2002) 176-182.

329. Pichini, S., I. Altieri, M. Pellegrini, R. Pacifici, and P. Zuccaro: Hair analysis for nicotine and cotinine: evaluation of extraction procedures, hair treatments and development of reference material; Forensic Sci. Int. 84 (1997) 243-252.

330. Kintz, P.: Gas chromatographic analysis of nicotine and cotinine in hair; J. Chrom. 580 (1992) 347-353.

331. Mizuno, A., T. Uematsu, A. Oshima, M. Nakamura, and M. Nakashima: Analysis of nicotine content of hair for assessing individual cigarette-smoking behaviour; Ther. Drug Monit. 15 (1993) 99-104.

332. Nilsen, T. and O.G. Nilsen: Accumulation of nicotine in human hair during long-term controlled exposure to 
a low concentration of nicotine vapour; Pharmacol. Toxicol. 81 (1997) 48-52.

333.Zahlsen, K. and O.G. Nilsen: Gas chromatographic analysis of nicotine in hair; Environ. Technol. 11 (1990) 353-364.

334. Dimish-Ward, H., H. Gee, M. Brauer, and V. Leung: Analysis of nicotine and cotinine in the hair of hospitality workers exposed to environmental tobacco smoke; J. Occ. Environ. Med. 39 (1997) 946-948.

335. Gerstenberg, B., G. Schepers, P. Voncken, and H. Völkel: Nicotine and cotinine accumulation in pigmented and unpigmented rat hair; Drug Metab. Dispos. 23 (1995) 143-148.

336. Dehn, D.L., D.J. Claffey, M.W. Duncan, and J.A. Ruth: Nicotine and cotinine adducts of a melanin intermediate demonstrated by matrix-assisted laser desorption/ionization time-of-flight mas spectrometry; Chem. Res. Toxicol. 14 (2001) 275-279.

337. C. Eliopoulos, J. Klein, and G. Koren: Validation of self-reported smoking by analysis of hair for nicotine and cotinine; Ther. Drug Monitor. 18 (1996) 532-536.

338. Uematsu, T., A. Mizuno, S. Nagashima, A. Oshima, and M. Nakamura: The axial distribution of nicotine content along hair shaft as an indicator of changes in smoking behaviour: elevation in a smoking-cessation programme with or without the aid of nicotine chewing gum; Br. J. clin. Pharmac. 39 (1995) 665-669.

339. Pichini, S., Ó. Garcia-Algar, L. Munoz, O. Vall, R. Pacifici, C. Figueroa, J.A. Pascual, D. Diaz, and J. Sunyer: Assessment of chronic exposure to cigarette smoke and its change during pregnancy by segmental hair analysis; J. Exposure Anal. Environ. Epidemiol. 13 (2003) 144-151.

340. Feldman, Y., G. Koren, D. Mattice, H. Shear, E. Pellegrini, and S.M. MacLeod: Determinants of recall and recall bias in studying drug and chemical exposures in pregnancy; Teratology 49 (1989) 37-45.

341. Kintz, P., B. Ludes, and P. Mangin: Evaluation of nicotine and cotinine in human hair; J. Forensic Sci. 37 (1992) 72-76.

342. Klein, J. and G. Koren: Hair analysis - a biological marker for passive smoking in pregnancy and childhood; Human Expt. Toxicol. 18 (1999) 279-282.

343. Hardee, G.E., T. Stewart, and A.C. Capomacchia: Tobacco smoke xenobiotic compound appearance in mother's milk and involuntary smoke exposures. I. Nicotine and cotinine; Toxicol. Lett. 15 (1983) 109-112.

344. Luck, W. and H. Nau: Nicotine and cotinine concentrations in the milk of smoking mothers: influence of cigarette consumption and diurnal variation; Eur. J. Pediatr. 146 (1987) 21-26.

345. Schiffman, M.H., N.J. Haley., J.S. Felton, A.W. Andrews, R.A. Kaslow, W.D. Lancaster, R.J. Kurman, L.A. Brinton, L.B. Lannom, and D. Hoffmann: Biochemical epidemiology of cervical neoplasia: measuring cigarette smoke constituents in the cervix; Cancer Res. 44 (1987) 3886-3888.

346. Jones, C.J., M.H. Schiffman, R. Kurman, P. Jacob III., and N.L. Benowitz: Elevated nicotine levels in cervical lavages from passive smokers; J. Publ. Health 81 (1991) 378-379.

347. McCann, M.F., D.E. Irwin, L.A. Walton, B.S. Hulka, J.L. Morton, and C.M. Axelrad: Nicotine and cotinine in the cervical mucus of smokers, passive smokers and nonsmokers; Cancer Epidemiol. Biomarkers Prev. 1
(1992) 125-129.

348. Poppe, W.A., R. Peeters,P. Daenens, P.S. Ide, and F.A. Van Assche: Tobacco smoking and the uterine cervix: cotinine in blood, urine and cervical fluid; Gynecol. Obstet. Invest. 39 (1995) 110-114.

349. Vine, M.F., C.J. Tse, P. Hu, and K.Y. Truong: Cigarette smoking and semen quality; Fertil. Steril. 65 (1996) 835-842.

350. Macaron, C.I., Z. Macaron, M.-T. Maalouf, and G. Khazaal: Cotinine in seminal fluids of smokers, passive smokers and nonsmokers; J. Med. Liban. 45 (1997) 46.

351. Balabanova, S., G. Bühler, E. Schneider, H.J. Boschek, and H. Schneiter: Über die Ausscheidung von Nikotin mit dem apokrinen und ekkrinen Schweiß bei Rauchern und Passiv-Rauchern [Nicotine excretion by the apocrine and eccrine sweat in smokers and passive smokers]; Hautarzt 43 (1992) 73-76.

352. Kintz, P., A. Henrich, V. Cirimele, and B. Ludes: Nicotine monitoring in sweat with a sweat patch; J. Chromatogr. B 705 (1998) 357-361.

353. Al-Delaimy, W.K., G.N. Mahoney, F.E. Speizer, and W.C. Willett: Toenail nicotine levels as a biomarker of tobacco smoke exposure; Cancer Epidemiol. Biomarkers \& Prev. 11 (2002) 1400-1404.

354. Garcia-Algar, Ó., O. Vall, J. Segura, J.A. Pascual, D. Diaz, L. Muñoz, P. Zuccaro, R. Pacifici, and S. Pichini: Nicotine concentrations in deciduous teeth and cumulative exposure to tobacco smoke during childhood; JAMA 290 (2003) 196-197.

355. Fukami, T., M. Nakajima, R. Yoshida, Y. Tsuchiya, Y. Fujiki, M. Katoh, H.L. MeLeod, and T. Yokoi: A novel polymorphism of human CYP2A6 gene CYP2A6*17 has an amino acid substitution (V365M) that decreases enzyme activity in vitro and in vivo; Clin. Pharmacol. Ther. 76 (2004) 519-527.

356. Jarvis, M.J., C. Feyerabend, A. Bryant, B. Hedges, and P. Primatesta: Passive smoking in the home: plasma cotinine concentrations in non-smokers with smoking partners; Tobacco Control 10 (2001) 368-374.

357. Martínez, M.E., M. Reid, R. Jiang, J. Einspahr, and D.S. Alberts: Accuracy of self-reported smoking status among participants in a chemoprevention trial; Prev. Med. 38 (2004) 492-497.

358. Goulay, S.G., N.L. Benowitz, A. Forbes, and J.J. McNeill: Determinants of plasma concentrations of nicotine and cotinine during cigarette smoking and transdermal nicotine replacement; Eur. J. Clin. Pharmacol. 51 (1997) 407-414.

359. Li, C.Q., R.A. Windsor, L. Perkins, R.L. Goldenberg, and J.B. Lowe: The impact on infant birthweight and gestational age of cotinine-validated smoking reduction during pregnancy; JAMA 269 (1993) 1519-1524.

Address for correspondence:

Anthony R. Tricker

Philip Morris Products S.A.

PMI Research and Development

CH-2000 Neuchâtel

Switzerland

E-mail: anthony.tricker@pmintl.com 